\title{
Origin of the turbulent spectra in the high-altitude cusp: Cluster spacecraft observations
}

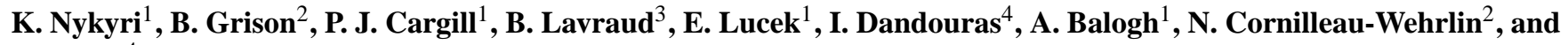 \\ H. Rème ${ }^{4}$ \\ ${ }^{1}$ The Blackett Laboratory, Imperial College, London, UK \\ ${ }^{2}$ CETP/CNRS-UVSQ, 10/12 Avenue de 1 Europe, 78140, Velizy, France \\ ${ }^{3}$ Los Alamos National Laboratory, Los Alamos, NM, USA \\ ${ }^{4}$ Centre d'Etude Spatiale des Rayonnements,31028 Toulouse Cedex 4, France
}

Received: 1 August 2005 - Revised: 21 February 2006 - Accepted: 28 March 2006 - Published: 19 May 2006

\begin{abstract}
High-resolution magnetic field data from Cluster Flux Gate Magnetometer (FGM) and the Spatio-Temporal Analysis of Field Fluctuations (STAFF) instruments are used to study turbulent magnetic field fluctuations during the highaltitude cusp crossing on 17 March 2001. Despite the quiet solar wind conditions, the cusp was filled with magnetic field turbulence whose power correlates with the field-aligned ion plasma flux. The magnetic field wave spectra shows power law behavior with both double and single slopes with break in the spectra usually occurring in the vicinity of the local ion cyclotron frequency. Strong peaks in the wave power close to local ion cyclotron frequency were sometimes observed, with secondary peaks at higher harmonics indicative of resonant processes between protons and the waves. We show that the observed spectral break point may be caused partly by damping of obliquely propagating kinetic Alfvén (KAW) waves and partly by cyclotron damping of ion cyclotron waves.
\end{abstract}

Keywords. Magnetospheric physics (Magnetopause, cusp and boundary layers; Plasma waves and instabilities) - Space plasma physics (Nonlinear phenomena; turbulence)

\section{Introduction}

The high-altitude cusps are regions where the magnetosheath plasma has the most direct access to the ionosphere, and their structure is determined by a complex interaction between the shocked solar wind and the geomagnetic field. The cusps are also a region of significant turbulence which can be created by solar wind (bowshock) perturbations, magnetic reconnection or intrinsic instabilities present in the cusp. Prior to the mid-1990s, in situ measurements of the cusp were relatively infrequent, although important results came from the HEOS

Correspondence to: K. Nykyri

(k.nykyri@ic.ac.uk) and Hawkeye missions (e.g. Paschmann et al., 1976; Kessel et al., 1996; Dunlop et al., 2000). The recent Polar and Interball missions have been important in illuminating many examples of cusp physics. Of particular interest for this paper has been the identification of lobe reconnection (Scudder et al., 2002; Fuselier et al., 2000; Russell et al., 2000), the spatial extent of the cusp (Zhou et al., 1999, 2000) and insitu magnetic field turbulence (Savin et al., 1998; Chen and Fritz, 1998; Le et al., 2001; Savin et al., 2002, 2004).

The four spacecraft, multi-instrument Cluster mission represents the first of a new generation of magnetospheric physics missions, and is ideal for studying the high- and mid-altitude cusps. Cluster has revealed for the first time many important aspects of the structure of the high-altitude cusps, and especially how they are related to the prevailing solar wind conditions (e.g. Lavraud and Cargill, 2005; Cargill et al., 2005; Lavraud et al., 2005). In particular, it is becoming apparent that the orientation of the Interplanetary Magnetic Field (IMF) plays a pivotal role. For Northward IMF, a picture is emerging of a cusp influenced by lobe reconnection (Vontrat-Reberac et al., 2003; Twitty et al., 2004) with Earthward-directed plasma jets being observed, but also containing regions of stagnant plasma (Lavraud et al., 2002, 2004). On the other hand, for Southward IMF, the cusp is dominated by tailward convection due to sub-solar reconnection (Cargill et al., 2004).

A particularly interesting aspect of the cusp encounters with Northward IMF have been observations of extensive regions of magnetic field turbulence seemingly associated with Earthward-directed plasma flows near the boundary between the cusp and lobe (Nykyri et al., 2003, 2004; Sundkvist et al., 2005). The level of turbulence appears to correlate with the magnitude of the flows and the plasma number flux, and also shows strong peaks at the ion cyclotron frequency. Both left and right-handed waves are seen: a puzzling observation is the presence of left-handed waves above the ion cyclotron frequency (also observed by Polar spacecraft,

Published by Copernicus GmbH on behalf of the European Geosciences Union. 
Le et al., 2001). The waves show no significant correlation between the spacecraft when the inter-spacecraft separation is either 600 or $100 \mathrm{~km}$ (Nykyri et al., 2003). It is unclear whether these waves are generated in-situ by the plasma flows, or whether they are convected from remote reconnection sites at the lobes. Since the reconnection site would be several $R_{E}$ away, this implies a damping time longer than $300 \mathrm{~s}$ for flows of $100 \mathrm{~km} / \mathrm{s}$. A recent study by Sundkvist et al. (2005) is implying a local generation mechanism for these waves: they observe that the Poyinting flux is changing direction at the proton gyrofrequency.

Our previous studies (Nykyri et al., 2003, 2004) were carried out using magnetic field data from the Flux Gate Magnetometer (FGM) instruments on Cluster (Balogh et al., 2001), and focused on the frequency range between $0.1 \mathrm{~Hz}$ to over $1 \mathrm{~Hz}$. For these examples, the proton cyclotron frequency $\left(f_{I C}\right)$ was in the range $0.7-1.5 \mathrm{~Hz}$. We found indications of wave power at higher cyclotron harmonics, and the transition to a different turbulence regime above $f_{I C}$. The latter possibility has also been discussed by Sundkvist et al. (2005), who suggest that the cusp waves belong to the kinetic Alfvén branch below the proton cyclotron frequency and are electromagnetic ion-Bernstein waves above that frequency. This is also confirmed by Grison et al. (2005) who used the $k$-filtering technique, and showed that kinetic Alfvén waves dominate the electromagnetic spectrum up to $1 \mathrm{~Hz}$ and above $0.8 \mathrm{~Hz}$ intense Bernstein waves are observed.

A limitation of our earlier studies was the level of instrument noise above a few $\mathrm{Hz}$ and so this paper returns to the topic of cusp turbulence by examining magnetic field turbulence at frequencies up to $10 \mathrm{~Hz}$ using measurements from the Cluster Spatio-Temporal Analysis of Field Fluctuation (STAFF) experiment (Cornilleau-Wehrlin et al., 1997; Cornilleau-Wehrlin et al., 2003) as well as from FGM. The main objective in using the two instruments is to get a continuous spectra from lower (FGM) to higher (STAFF) frequencies which enables us to study spectral structure both below and above the local ion cyclotron frequency $\left(f_{I C}\right)$.

We return in this paper to the well-studied high-altitude cusp crossing on 17 March 2001 (Vontrat-Reberac et al., 2003; Nykyri et al., 2003, 2004). Section 2 presents the relevant instrumentation and cusp overview, Sect. 3 the analysis techniques, Sect. 4 the measurements and Sects. 5 and 6 our interpretation of the observed spectral structure in terms of wave damping.

\section{Instrumentation and overview of cusp}

We use data from three instruments on-board Cluster. High resolution (22.4 vectors/s) magnetic field measurements are obtained from the Flux Gate Magnetometer (FGM: Balogh et al., 1997, 2001) from all four spacecraft. FGM data provides the overall cusp magnetic field context, and wave power at frequencies up to $3 \mathrm{~Hz}$ can be accurately calculated.
Search coil magnetometers (STAFF: Spatio-Temporal Analysis of Field Fluctuation experiment: Cornilleau-Wehrlin et al., 2003; Cornilleau-Wehrlin et al., 1997) on-board all four spacecraft are used to calculate wave power from $1 \mathrm{~Hz}$ up to $10 \mathrm{~Hz}$. Ion plasma measurements are obtained using the Cluster Ion Spectrometry (CIS: Rème et al., 2001) experiment and are essential for providing the plasma context. Data from the Hot Ion Analyser (HIA) instrument on-board spacecraft 1 and 3 and the ion COmposition and DIstribution Function analyser (CODIF) instrument on-board spacecraft 4 are used. The plasma velocity moments are available every $\sim 4 \mathrm{~s}$ from spacecraft 1 and 4 and every $12 \mathrm{~s}$ from spacecraft 3 .

The cusp crossing chosen occurs on an outbound orbit between 05:00 and 07:00 UT on 17 March 2001. This interval has been extensively studied (Vontrat-Reberac et al., 2003; Nykyri et al., 2003, 2004), so that the overall phenomenology is well established. Figure 1 shows the power spectral slopes (discussed later in Sects. 4.3 and 4.4) and plasma parameters measured by spacecraft 1, 3 and 4 between 05:00-06:00 UT. The color code used in this paper is as follows: black, red, green and blue correspond to spacecraft 1,2, 3 and 4 respectively (referred to hereafter as sc1, sc2, sc3, sc4). Panels b-f show the ion beta $(\beta)$, plasma density $(N)$, parallel plasma velocity $\left(V_{\|}\right)$, Alfvén Mach number $\left(M_{A}\right)$ and the angle between plasma flow and the magnetic field $\left(\theta_{B V}\right)$. The spacecraft separation is $600 \mathrm{~km}$ and Cluster altitude in the cusp is $\sim Z_{G S M}=8 R_{E}$.

The spacecraft leave the magnetosphere shortly after 05:00 UT, and cusp entry can be seen first as an enhanced density at sc3 (Fig. 1c). Between 05:07 and 05:13 UT, the spacecraft sequentially entered the cusp, a region characterized here by a density of a few particles $\mathrm{cm}^{-3}$ with an Earthward-directed plasma flow of approximately $250 \mathrm{~km} / \mathrm{s}$ (Fig. 1d), although this value varies between the spacecraft.

Cluster remains in the cusp from approximately $05: 13$ to 06:20 UT, and during this time the flows decline to almost zero. However, this decline masks considerable structure, with strong velocity shears being present over timescales of a few minutes. Between 05:00-06:00 UT the magnetic field gradually decreases (not shown) from $\sim 100 \mathrm{nT}$ to $45 \mathrm{nT}$, implying proton cyclotron frequencies of $1.5-0.7 \mathrm{~Hz}$. In this paper we only focus on data up to 06:00 UT.

The solar wind conditions (not shown) are obtained from Advanced Composition Explorer (ACE) spacecraft at the L1 point (a lag time was applied assuming $285 \mathrm{~km} / \mathrm{s}$ solar wind speed). During the interval of 04:30-06:30 UT solar wind had a magnetic field magnitude of approximately $5 \mathrm{nT}, B_{z}$ was mostly pointing northward (except for a brief southward turning at about 05:40 UT), with a magnitude of approximately $3 \mathrm{nT}$. $B_{y}$ was mostly positive with a magnitude of $3 \mathrm{nT}$ but turned negative at about 05:17 UT for about $35 \mathrm{~min}$. $B_{x}$ was close to zero until $\sim 5: 17$ UT at Cluster after which it turned to $\sim 2.5 \mathrm{nT}$. 


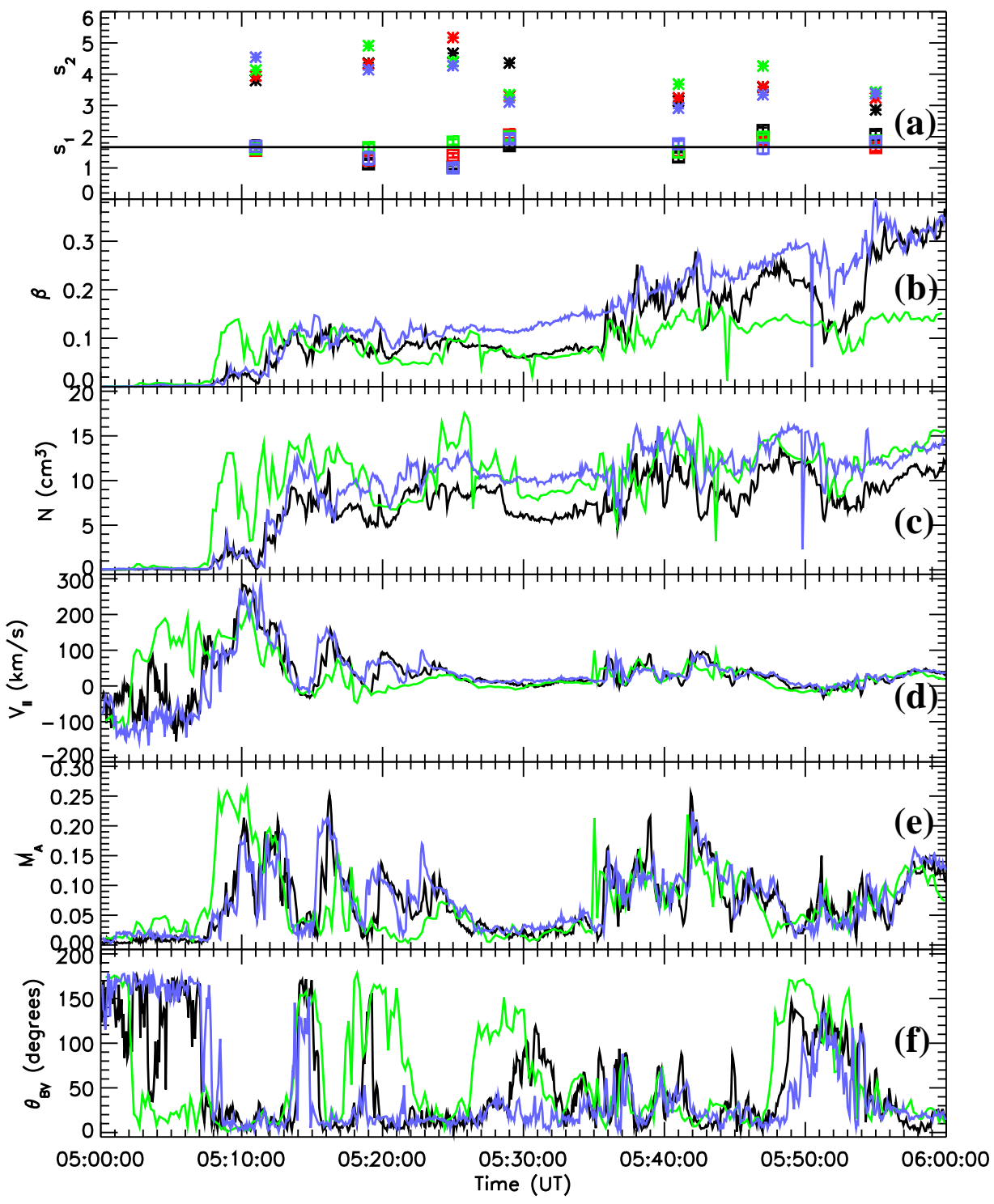

Fig. 1. Power spectral slopes and plasma parameters between 05:00-06:00 UT. Panel (a) shows inertial $\left(s_{1}\right)$ and dissipation $\left(s_{2}\right)$ range slopes. The solid line is at 5/3. Panels (b)-(f) show the ion plasma beta $(\beta)$, the plasma density $(N)$, parallel plasma velocity $\left(V_{\|}\right)$, Alfvén Mach number $\left(M_{A}\right)$ and the angle between plasma flow and the magnetic field $\left(\theta_{B V}\right)$. Black is data from spacecraft 1 (hereafter sc1), red from sc2, green from sc3 and blue from sc4. The spacecraft separation is $600 \mathrm{~km}$ and Cluster altitude in the cusp is $\sim Z_{G S M}=8 R_{E}$.

In analyzing the magnetic field turbulence we use same temporal demarcation of the cusp as described in Nykyri et al. (2004) who divided the analysis into regions based on the local plasma properties: a strong shear-flow cusp between 05:07 and 05:25; a stagnant cusp between 05:25 and 05:35 and a moderate shear-flow cusp between 05:35 and 05:56.

\section{Analysis methods of cusp magnetic field fluctuations}

We evaluated the total power of the magnetic field fluctuations between 05:00-06:00 UT using data from both the
STAFF and FGM instruments. The magnetic field time series was examined and the short duration data gaps were linearly interpolated. Before evaluating the Fast Fourier Transform (FFT) for each interval, a Hanning window was applied to the de-trended data set. The total power is then the trace of the power spectral matrix: $P_{t o t}=P_{x}+P_{y}+P_{z}$.

3.1 Combining STAFF and FGM: waves close to the ion cyclotron frequency

In order to ensure that the spectra from the two instruments can be combined we have checked that in the instrumental 


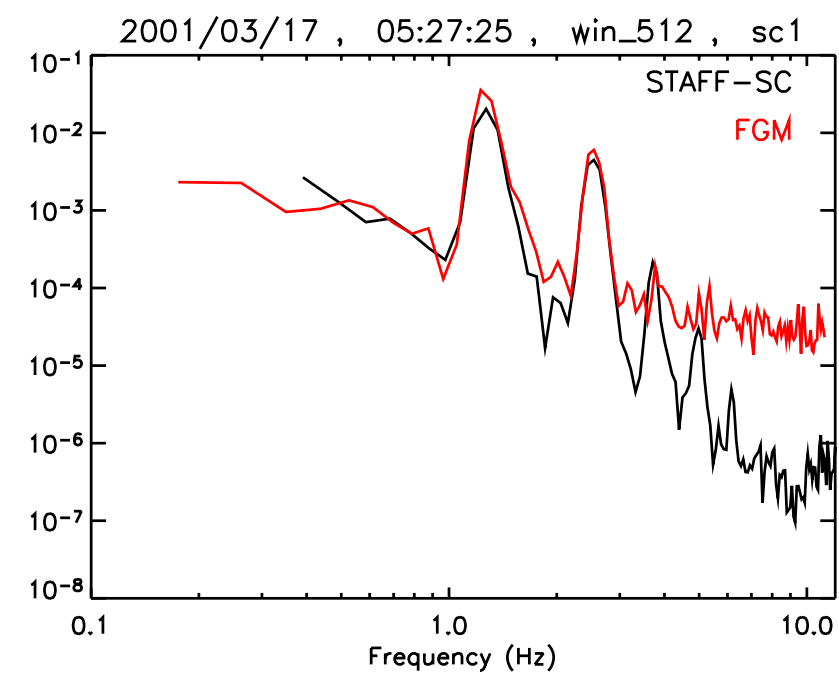

Fig. 2. Total power of magnetic field fluctuations observed by Cluster FGM (red) and STAFF (black) instrument on-board of spacecraft 1 centered at 05:27:25 UT $(2 \times 256$ data points are used $)$.

overlap region $(\sim 0.5-2 \mathrm{~Hz})$ both instruments measure the same power. For this purpose 512 point windows $(\sim 22.9 \mathrm{~s})$ were used at each interval of the comparison study in Sect. 4.3.

As an example of how FGM and STAFF data can be combined, we first look at the strong spectral peaks near the ion cyclotron frequency and its harmonics when the spacecraft are in the stagnant cusp. Examination of the magnetic field time series shows that the several small amplitude $(\sim 1-2 \mathrm{nT})$ wave trains were observable at all four spacecraft between $\sim 05: 25-05: 29$ UT and between $\sim 05: 45-05: 49$ UT, showing no correlation between the spacecraft pairs (see Nykyri et al. (2004) for more details).

Figure 2 shows the total power in the magnetic field fluctuations centered at 05:27:25 UT (for each instrument the data is averaged over 2 windows of 256 points) measured by FGM (red) and STAFF (black) on spacecraft 1. It is clear that between $0.5-2.8 \mathrm{~Hz}$, the power from both instruments are in good agreement. The frequency corresponding to the dominant peak is about 1.1 times the local ion cyclotron frequency. The first harmonic of the fundamental frequency is also observable from both FGM and STAFF. Because of the better sensitivity at higher frequencies, STAFF can also detect the 2nd, 3rd and 4th harmonics (see also Cargill et al., 2005).

Thus, both instruments measure the same peak of the fundamental frequency where their measurements overlap, so we have confidence in combining their spectra when using longer (approximately $6.1 \mathrm{~min}$ ) intervals.

\subsection{Slope validation}

However, longer window sizes have better statistics and are used to evaluate the slope of the power spectra (Sect. 4.3). There we use $\sim 6.1$ min windows for both instruments which corresponds to 8192 points with FGM sampling frequency of 22.4 vectors/s. Because the sampling frequency for STAFF is slightly higher than for FGM, we require more STAFF points to give the same total time interval. However, instead of a single window it is sometimes better to use short but numerous windows for STAFF spectra.

The magnetic field power spectra shows a clear break during some intervals dividing the spectra in two different power law regimes. We define the frequency at which this dispersion in spectrum occurs as a break point frequency, $f_{b f}$. For each interval, we have visually determined the $f_{b f}$ and the frequency range of the slope calculation for both instruments. FGM data are used below the dispersion breakpoint (typically below $1 \mathrm{~Hz}$ ) and STAFF-SC above it (up to $10 \mathrm{~Hz}$ ). We illustrate this point in Fig. 3c (the rest of Fig. 3 will be discussed fully in Sect. 4.3), which shows the total power of magnetic field fluctuations measured by sc3 from FGM (red) and STAFF (black) instrument at an interval centered at 05:19 UT. The breakpoint is visually determined to be at $1 \mathrm{~Hz}$ which is indicated by a black vertical line. During this interval the FGM data is used in slope calculation between $0.015-0.15 \mathrm{~Hz}$ and STAFF data is used between $3-10 \mathrm{~Hz}$. It can be seen that both instruments agree between $0.6-3 \mathrm{~Hz}$.

In addition to these studies, the slopes have been evaluated over a common range of frequencies (between 0.5 and $2 \mathrm{~Hz}$ ) from both instruments. These slopes do not have a physical meaning but they indicate that the spectral breaks are not an instrumental effect. Table 1 contains a comparison of the slopes estimated from STAFF and FGM in the frequency range $[0.5,2 \mathrm{~Hz}]$ for intervals centered at 05:41:00 UT. The comparison of the results obtained from one large-window FFT and the mean of many short windows cannot be made when spectral peaks are detected because of the number of frequencies present in the peak (512 point windows have a low resolution in frequency). So, the comparison has been performed during an interval that displays no peaks, and where almost no spectral break is detected (see Fig. 14a, which will be fully explained in Sect. 6).

The upper part of the table presents results obtained using a single FFT window for STAFF, whereas the lower table uses the mean of 18 consecutive 512 point FFTs (avoiding data gaps). The difference of the duration between the FGM 8192 point window and the 9216 point STAFF window is less than $1 \%$. The presence of data gaps in the STAFF data induces large errors in the spectrum at low frequency and also makes the slope steeper. On the contrary, the use of 512 point windows gives results similar to those obtained from FGM. When no data gaps are present (e.g. sc1), the use of one large window or 18 shorter windows is equivalent. Thus it is better to use short but numerous windows for STAFF spectra. 

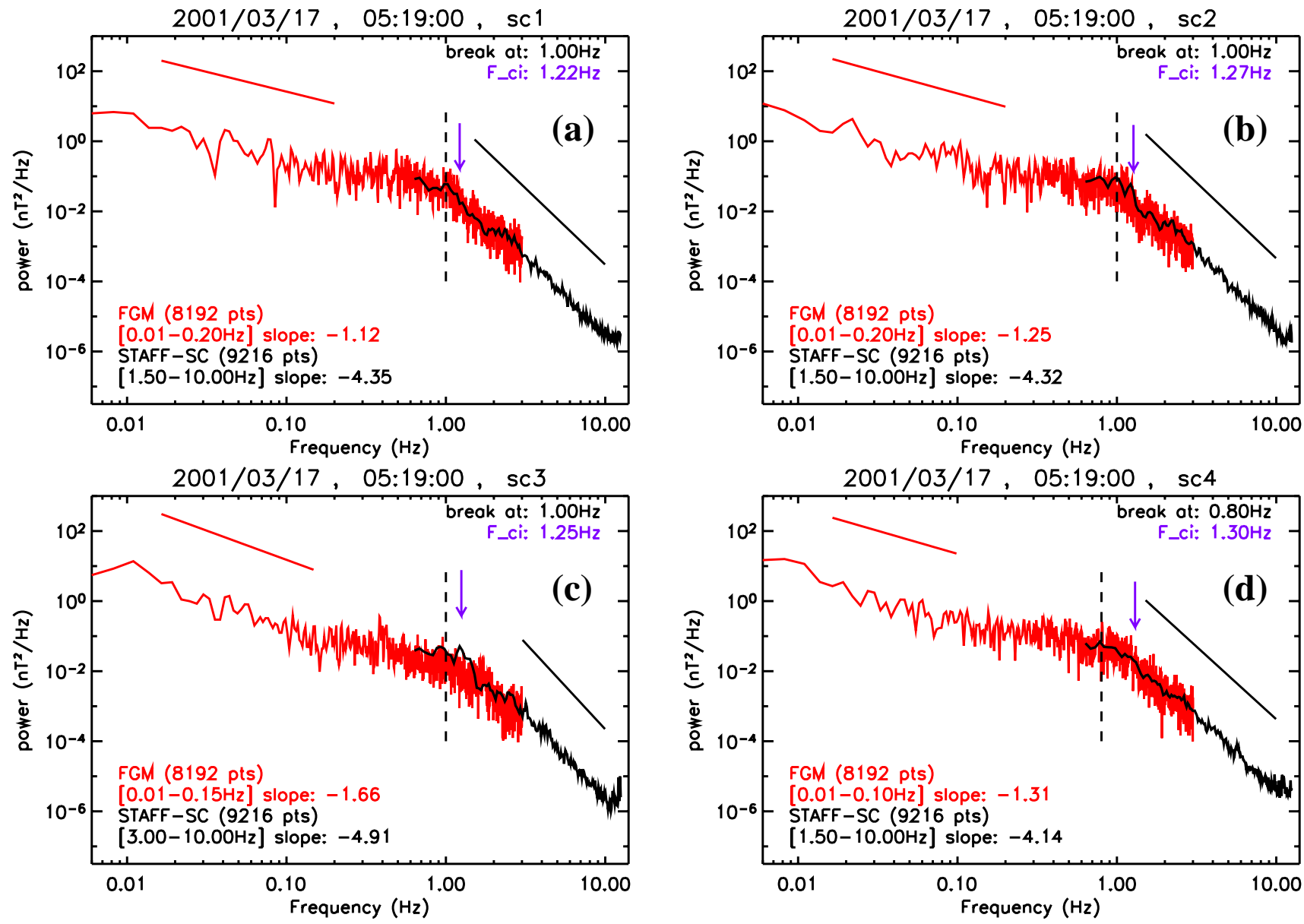

Fig. 3. The power spectrum of magnetic field fluctuations observed by Cluster FGM (red) and STAFF (black) instrument at interval centered at 05:19 UT by sc1 (a), sc2 (b), sc3 (c), and sc4 (d). Power law slopes are shown above the spectra, and their methods of evaluation are discussed fully in the text. The spectral breakpoint is marked with black vertical line and local $f_{I C}$ with purple arrow.

Thus the agreement between slope measurements from FGM and STAFF data during the same time interval and in the same frequency range ensure us that the spectral breaks do not originate from instrumental artifact but have a physical meaning.

\subsection{Stationarity of the time series}

In order to assess the stationarity of the time series, we have computed the variance of the plasma velocity field at each spacecraft for the same time intervals as the slope calculation. Figure $4 \mathrm{a}$ shows the velocity field variance using a $\sim 6.1$-min window and the total plasma velocity measured by sc1, sc 3 and sc 4 is shown in Fig. $4 \mathrm{~b}$. The variance is less than $45 \mathrm{~km} / \mathrm{s}$ for all the intervals after 05:17 UT, which is less than $10 \%$ of the local Alfvén speed (Fig. 2c). Because the Alfvén/ion cyclotron modes have phase speeds of $v_{p h}=v_{A} \cos \theta_{k B}$, where $\theta_{k B}$ is the angle of propagation with respect to magnetic field, for most cases the waves will
Table 1. Slopes of power spectra between [0.5-2 Hz] at 5:41:00 UT.

\begin{tabular}{lllll}
\hline & $\begin{array}{l}\text { FGM } \\
(8192 \text { pts. })\end{array}$ & $\begin{array}{l}\text { STAFF } \\
(9216 \text { pts. })\end{array}$ & FGM-STAFF & gaps \\
\hline Sc1 & -3.18 & -3.32 & 0.14 & none \\
Sc2 & -2.46 & -3.93 & 1.47 & many \\
Sc3 & -2.38 & -2.53 & 0.15 & small \\
Sc4 & -2.6 & -3.89 & 1.29 & small \\
\hline & FGM & STAFF & FGM-STAFF & gaps \\
& $(8192$ pts. $)$ & $(18 \times 512$ pts. $)$ & & \\
\hline Sc1 & -3.18 & -3.31 & 0.13 & none \\
Sc2 & -2.46 & -2.4 & -0.06 & two \\
Sc3 & -2.38 & -2.58 & 0.2 & one \\
Sc4 & -2.6 & -2.56 & -0.04 & one \\
\hline
\end{tabular}




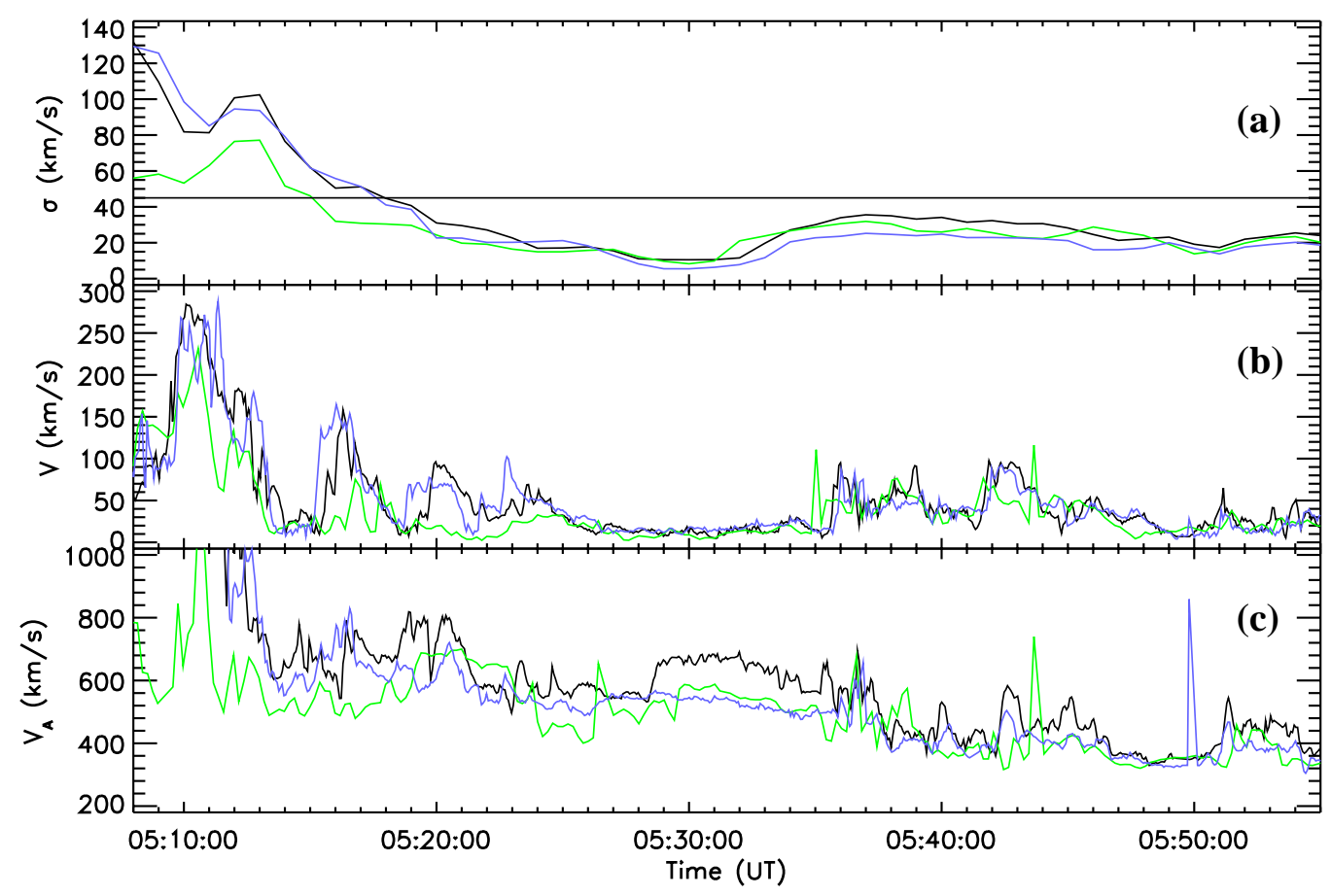

Fig. 4. Variance $(\sigma)$ of the plasma velocity shown (a) during each $\sim 6.1$-min interval used in the power spectral slope calculations. The total plasma velocity (b) and the Alfvén speed (c) measured by sc1, sc3 and sc4 between 05:00-06:00 UT are shown.

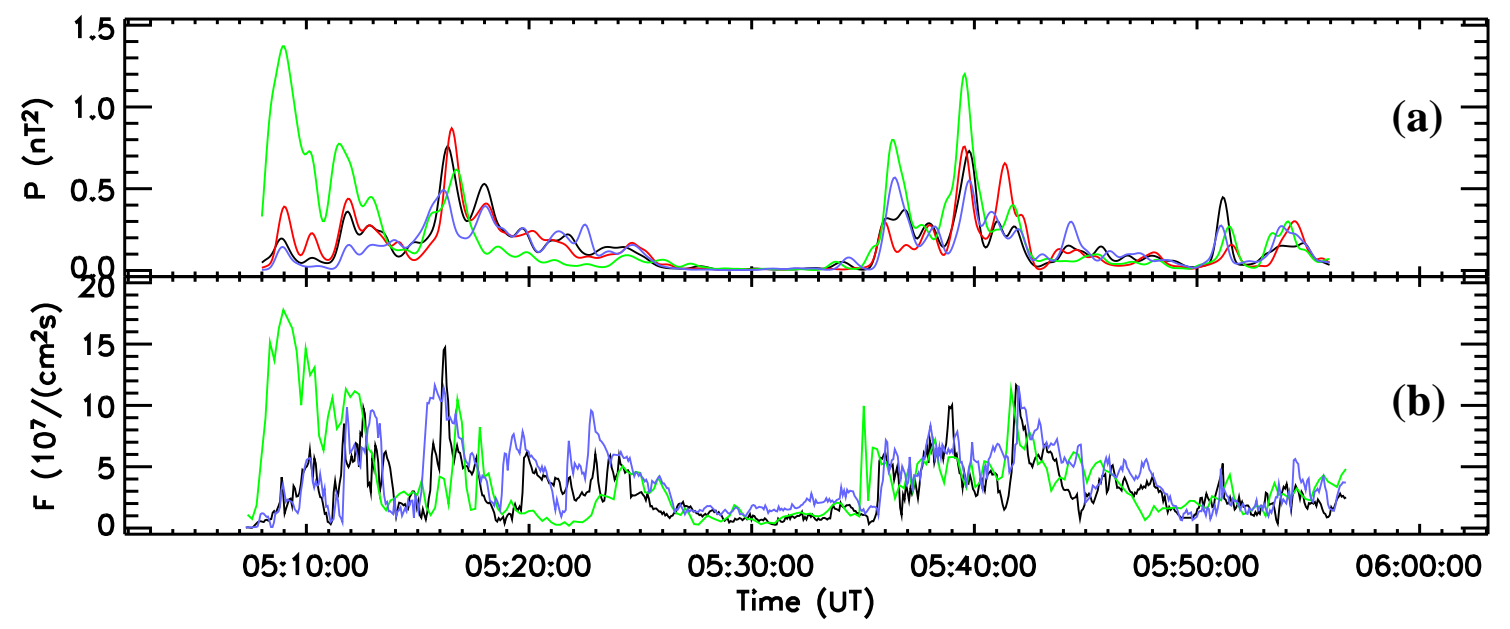

Fig. 5. Panel (a) shows the total integrated power in the magnetic field fluctuations observed by the FGM instruments on all four spacecraft between 05:00-06:00 UT. The frequency range is $0.06 \mathrm{~Hz}-3 \mathrm{~Hz}$ and a 2048 point window shifted with a 4-s increment is used). Panel (b) shows the total ion plasma number flux measured by sc1, sc3 and sc4.

propagate faster than the flows, and the intervals centered after 05:17 UT may be considered reasonably steady.

In addition to the intervals used in the comparison study we have also evaluated FGM spectra with 1024, 2048, 4096, and 16384 points corresponding to $45.4,90.7,182.9$ and $731.5 \mathrm{~s}$, respectively. Although there are intervals before 05:17 UT where the variance is less than $45 \mathrm{~km} / \mathrm{s}$ using the 90.7-s interval, errors in the power law indices increase for such short intervals. With a 182.9-s ( $\sim 3 \mathrm{~min})$ interval the variance shows little change, but the errors in slopes are again larger. Longer $\sim 12$-min windows are used to check the results of the 6.1-min slope calculations during intervals when the spectral breakpoint was observed at lower frequencies. Thus, the 6.1-min windows (8192 FGM points) give the best estimates for spectral indices. 


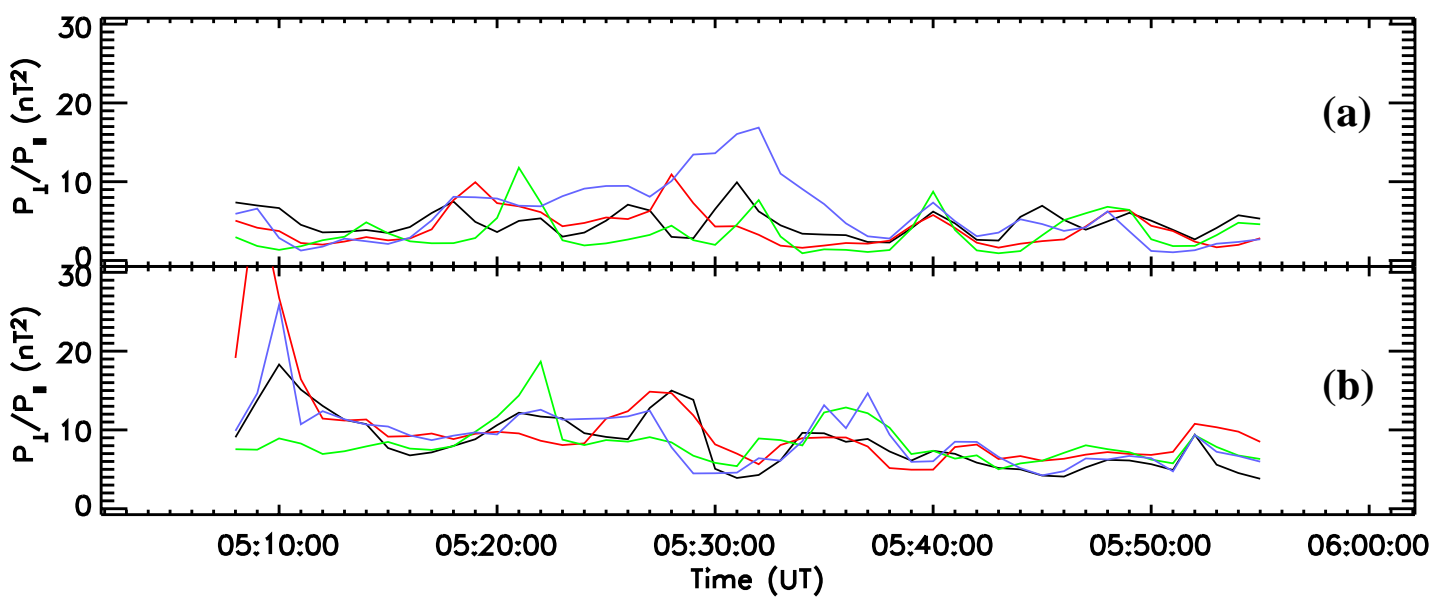

Fig. 6. The ratio of the integrated perpendicular and parallel (with respect to the magnetic field) power, $P_{\perp} / P_{\|}$, at the inertial range (a) between $0.015 \mathrm{~Hz}-f_{b f}$ and at the range between $f_{b f}-3.0 \mathrm{~Hz}$ (b). Calculations use 8192 point window shifted by 1-min increments over FGM data.

\section{Observations}

\subsection{Total integrated power of magnetic field fluctuations}

To set our investigation in context, we present the time history of the magnetic field fluctuations. Figure 5a shows the integrated power in magnetic field fluctuations between 05:00 and 06:00 UT measured by FGM with a 2048 point window ( $~ 93.1 \mathrm{~s})$ sequentially shifted with a 4-s increments through the cusp. The integration is performed over a frequency range of $0.06 \mathrm{~Hz}-3 \mathrm{~Hz}$ (The upper limit of $3 \mathrm{~Hz}$ is chosen to avoid the lower sensitivity of FGM at higher frequencies). Figure $5 \mathrm{~b}$ shows the ion number flux, $f=n v_{t o t}$, (or ion current $J_{i} / q$ ) measured by sc1, sc3 and sc4. One can see that the integrated power of the magnetic field fluctuations correlates well with the ion number flux. (Grison et al. (2005); Sundkvist et al. (2005) also show the correlation between wave activity and field-aligned ion fluxes). This is especially clear at cusp entry 05:07:30 UT, where sc1 and sc4 measure a low flux while also seeing a low level of magnetic field fluctuations, while sc3 sees an increased flux. The total plasma velocity (see Fig. 4b) and field-aligned plasma velocity (see Fig. 1d) at cusp entry is similar for all 3 spacecraft but sc3 sees an enhanced plasma density (Fig. 1c) compared to other two which results in enhanced plasma number flux. When the flow and number flux vanish before 05:30 UT, so does much of the power in the waves (see Fig. 2. and also Cargill et al. (2005); Grison et al. (2005)). Smaller scale velocity fluctuations re-appear at about 05:35 UT and persist throughout the rest of the interval.

\subsection{Transverse vs. compressional fluctuations}

Using FGM data we have evaluated the ratio of the perpendicular and parallel (with respect to the ambient magnetic field) wave power, $P_{\perp} / P_{\|}$, where $P_{\perp}=P_{t o t}-P_{\|}$and $P_{\|}$is the power in mean field $(|\mathbf{b}|)$ fluctuations. Figure 6 shows the ratio of the integrated perpendicular and parallel wave power, $P_{\perp} / P_{||}$between $0.015 \mathrm{~Hz}$ and break frequency $\left(f_{b f}\right)$ (a), and between $f_{b f}$ and $3.0 \mathrm{~Hz}$ (b). An 8192 point window slided with 1-min increments through the data set between 05:00-06:00 UT is used. In both ranges the fluctuations are mostly transverse.

\subsection{Calculation of power spectral indices below and above spectral breakpoint}

We have evaluated the power spectra obtained from FGM and STAFF data from all four spacecraft using $~ 6.1$-min intervals, centered at seven different times: 05:11 (cusp entry) (However, we note that this interval has a problem of stationarity as described in Sect. 3.3), 05:19 (shear-flow cusp), 05:25 (stagnant cusp boundary), 05:29 (stagnant cusp), 05:41 (moderate shear flow cusp), 05:47 (stagnant cusp boundary) and 05:55 UT (moderate shear flow cusp). In this section we first discuss in detail one of the seven periods, that centered at 05:19 UT, then review the others.

As an example of the power spectra, Figs. 3a-d show the total power in the magnetic field fluctuations measured by spacecraft $1-4$ respectively during intervals centered at 05:19 UT. The black curve and the regression line correspond to STAFF data and the corresponding red ones to FGM data. One can clearly distinguish two slopes of the power spectra. Such a double slope spectra in the cusp has also been reported from Interball-1 and Polar observation (Savin et al., 2002) as well as from Cluster observations (Sundkvist et al., 2005). In the solar wind such a double slope magnetic field spectra has been interpreted in terms of an inertial range and dissipation range (i.e. Goldstein et al. (1994); Leamon et al. (1998) and references therein). The solar wind 
has the same $-5 / 3$ power law in the inertial range as a high Reynolds number fluid (Kolmogoroff, 1941). In the solar wind the plasma flow speed is much larger than the typical wave speed $\left(V_{S W} \gg V_{\text {phase }}\right)$, so that a time series can be interpreted as a 1-D spatial cut, enabling a transition from the frequency space to the wave number $(k)$ space. However, in the high-altitude cusp (and for the presented crossing) the Alfvén speed and thus the phase speed of the waves is much larger than the plasma flow speed ( $V_{\text {phase }} \gg V$ ), so that one cannot do a direct transition from $f$-space to the $k$-space. The magnetic field power spectrum is thus likely to be dominated by propagating temporal fluctuations (waves) from the different sources. In the regions of enhanced flows the frequency of these waves is Doppler-shifted according to

$f_{S C}=\frac{1}{2 \pi}\left(V k \cos \left(\theta_{k V}\right)+\omega\right)$,

where $k$ is the wavenumber and $\theta_{k V}$ is the angle between the $k$-vector and the plasma velocity. It is also possible that some Doppler shifted spatial structures are present. In Sect. 5 we will discuss possible interpretations for this double sloped spectra. However, for simplicity we will refer hereafter to the spectra below the spectral breakpoint (usually at $f_{I C}$ ) as an "inertial" range and above as a "dissipation" range, although they cannot be directly compared with the existing turbulent theories of the solar wind.

The frequency ranges used in the slope calculations in the inertial and dissipation ranges are visually determined based on the shape of the spectra. During this interval the upper frequency limit for the inertial range is below $0.2 \mathrm{~Hz}$ which gives slopes of $-1.12,-1.25,-1.66$ and -1.31 with error-bars of $25 \%, 24 \%, 15 \%$, and $32 \%$ for sc1, sc2, sc 3 and sc 4 , respectively. Here the spacecraft separation is $600 \mathrm{~km}$ and we did not observe any correlation of the magnetic field time series between different spacecraft pair. Therefore we cannot apply the $k$-filtering technique (Sahraoui et al., 2003; Grison et al., 2005) (which works if the wave length of the waves is larger than the spacecraft separation) to do a transition from the frequency space to the $k$-space.

The slopes calculated from STAFF data above $1 \mathrm{~Hz}$ are much steeper and for this time interval vary between -4.9 and -4.1 , such slopes being characteristic of a turbulent dissipation range (see (Goldstein et al., 1994) for a discussion of this in the solar wind). In each case, the spectral breakpoint of $\sim 1 \mathrm{~Hz}$ is slightly below the local proton cyclotron frequency, which is marked with purple arrow in Fig. 3.

This analysis can be extended to other intervals with the aim of investigating whether there is any connection between the values of the indices and the local plasma properties, especially the velocity. Figure 1a shows the magnitudes of inertial, $s_{1}$, (squares) and dissipation range, $s_{2}$, (stars) power law slopes with the associated error bars (these are smaller than the size of the square) during the seven intervals described in the beginning of this section.
Figure 13a shows the magnitudes of inertial range slopes with error bars (the range of slope calculation is visually determined for each interval) determined from the FGM for each minute through the cusp. The solid, horizontal line is at $5 / 3$, and the solid wavy line marks the average slope value of all four spacecraft at each interval. There are significant differences between different times, and between different spacecraft at the same time.

When considering all intervals, the inertial range slopes vary from -1.0 to -2.7 . In the dissipation range, we find slopes between -2.9 and -5.2 . The exact frequency range for slope calculation for both instruments is determined individually for each spacecraft based on the shape of the spectra as explained in Sect. 3.2.

\subsection{Comparison of spectral indices with the plasma param- eters}

As Cluster moves through the cusp, not only do the plasma parameters change, but so does the background magnetic field. The local Alfvén speed decreases from about $800 \mathrm{~km} / \mathrm{s}$ to $350 \mathrm{~km} / \mathrm{s}$ between 05:13 and 06:00 UT (see Fig. 4c) and during each 6.1-min interval Cluster moves approximately $1100 \mathrm{~km}$ in a Sunward direction. After 05:12 UT the ion temperature (not shown) is almost constant at all four spacecraft, and the density increases as shown in Fig. 1c, albeit with fluctuations. In order to compare the plasma parameters with spectral indices at each interval we have calculated $\sim 6.1$-min averages of the plasma parameters, their maximum and minimum value and the difference between these maximum and minimum value during each interval (this is done for those parameters shown in Fig. 1 but also for others such as perpendicular velocity, parallel number flux, total number flux, perpendicular number flux, temperature, plasma pressure and total pressure). No correlation with the power spectral slopes was found in either inertial or dissipation ranges.

\subsection{Ion distribution functions and plasma velocity during wave observations}

The plasma velocity shows significant changes in this interval. Between 05:07 and 05:25 UT the field-aligned velocity component (Fig. 1d) occasionally differs between the spacecraft, indicative of a velocity shear with a scale of $600 \mathrm{~km}$. These field-aligned flows can be interpreted as a signature of lobe reconnection tailward of the cusp (VontratReberac et al., 2003). Figures $7 \mathrm{a}-\mathrm{c}$ show ion distribution functions in the parallel-perpendicular velocity plane during 3 intervals measured by HIA on sc1 (Fig. 7a) and CODIF on sc4 (Figs. 7b-c). At 05:15:21 UT sc4 encounters a fieldaligned flow of $\sim 150 \mathrm{~km} / \mathrm{s}$ (Fig. 1d) and measures a Dshaped shell (horseshoe) distribution (Fig. 7c), characteristic of a recently reconnected field line (Cowley, 1982). These shell distributions are known to be unstable to the generation of electrostatic Ion Bernstein (IB) waves (Janhunen et al., 

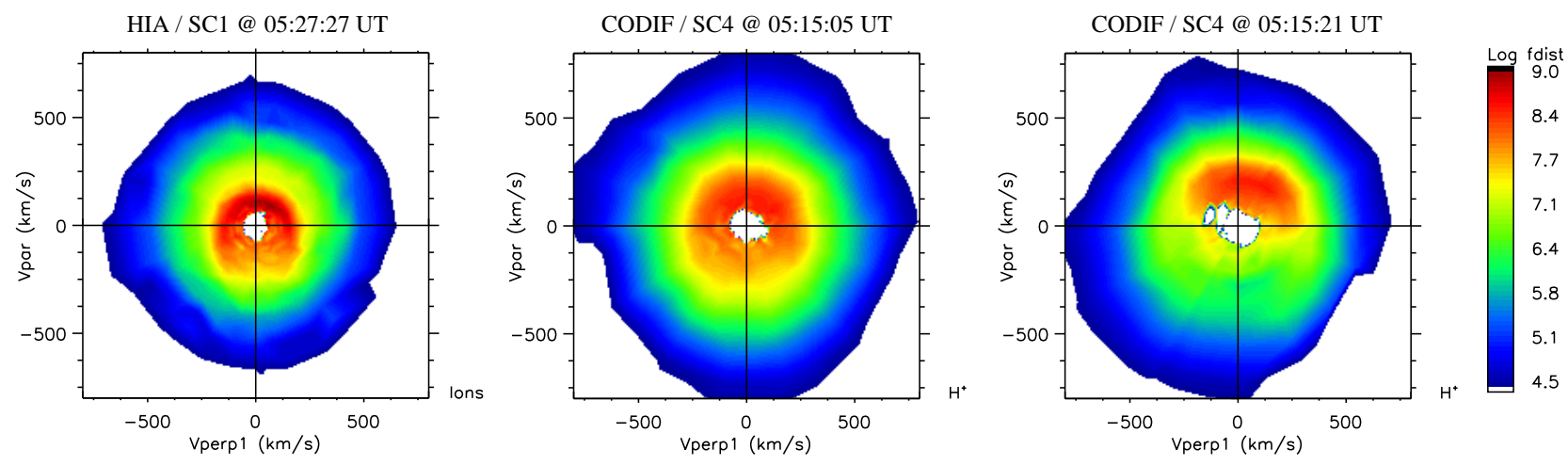

Fig. 7. Ion distribution functions in parallel/perpendicular-plane (from HIA-instrument) at 05:27:27 UT (a). Proton distribution functions (from CODIF-instrument) at sc4 (at 05:15:01 UT (b) and at 05:15:21 UT (c)), respectively.

2003 ) and to the electromagnetic ion cyclotron waves (Sundkvist et al., 2005). Figure 7a of Nykyri et al. (2004) shows that sc 4 also observes waves close to the ion cyclotron frequency between 05:15:29-5:15:41 UT, but only the fundamental peak is clear (with FGM) and the waves do not form as regular wave train as at $\sim 05: 27: 27 \mathrm{UT}$. The STAFF $b_{x}$ spectra at sc4 between 05:15:35-05:15:45 UT shows two peaks: one at the cyclotron frequency at $\approx 1.3 \mathrm{~Hz}$ and other at $\approx 3 \mathrm{~Hz}$, so that the second peak is not a harmonic. Prior to this interval sc 4 observes an isotropic distribution at 05:15:05 UT (Fig. 7b) corresponding to a diminished field-aligned flow (Fig. 1d). There is also an extensive literature concerning the generation of IC waves due to instabilities in plasma flows, especially for ionospheric parameters (e.g. Kindel and Kennel, 1971; Penano and Ganguli, 2002). For field-aligned flow (such as we mostly detect here (see Fig. 1d and 1f), only electrostatic calculations have been carried out, but they suggest that waves can be excited at many harmonics of the $\Omega_{i}$ (Gavrishchaka et al., 2000; Ganguli et al., 2002). However, electrostatic is just a limiting case. Because the ion cyclotron perturbations are two dimensional they will always be associated with current and hence magnetic fluctuations. Transverse flow layers can also become unstable and excite IB waves (Reynolds and Ganguli, 1998).

The diminution of the flow magnitude, especially after 05:25 UT, has two possible explanations. First, the ACE data shows that the IMF $B_{z}$ slightly rotates (and also the $B_{y}$ ), turning negative at about 05:30 UT, so that the amount of the reconnecting flux tailward of the cusp decreases, resulting in a weaker flow. Indeed parallel flows (with a lower magnitude) reappear after the IMF turns northward at about 05:35 UT.

Secondly, Cluster is moving further into the cusp, encountering field lines that have reconnected some time in the past. This can be seen as a more symmetric ion distribution function measured by sc 1 at 05:27:27 UT (Fig. 7a). This distribution coincides with the magnetic field observations of the ion cyclotron harmonics at 05:27:14-05:27:37 UT (Fig. 2). One can notice that the parallel part of the distribution (beam) is slightly enhanced, and in the other direction a small depletion can be seen, reminiscent of a small loss cone. The magnetic hodograms calculated by Nykyri et al. (2004) (Figs. 10 and 13) show that the waves are clearly electromagnetic at $\sim 05: 25-05: 29$ UT and 05:45-05:49 UT during the observations of the several harmonics. It is presently not clear why the harmonics are not clearly evident during the strongest velocity shear (05:15:21 UT) but favor the regions where plasma has become nearly stagnant (and has small velocity shear $10-40 \mathrm{~km} / \mathrm{s}$ ) and has these more symmetric distribution functions. However, they are not present in the regions where the plasma has been stagnant for several minutes (i.e. 05:30 UT).

\section{The origin of the spectral breakpoint}

There are a few possible ways to interpret the structure of the spectra presented in Fig. 3 and during other intervals. One is in terms of an "inertial" and a "dissipation" range. The examination of the magnetic field time series during each of these intervals shows the existence of several temporal scales and amplitudes (broadband spectra). Structures look mostly incoherent and random, but occasionally there are short duration intervals when more sinusoidal, clearly polarized discrete wave trains are observed "riding the lower frequency waves". Each of the 6.1-min spectra is therefore likely to be composed of a continuous broad band (power law) spectra as well as of discrete wave modes (peaks) superimposed on this spectra during some intervals. In MHD, oppositely propagating fluctuations can give rise to a turbulent energy cascade due to the non-linear interactions (Kraichnan, 1965). If a strong magnetic field is applied, the cascade is suppressed in the direction of the magnetic field. The Cluster trajectory is perpendicular to the ambient magnetic field direction, so that the observed broad band spectra may be a result of the turbulent cascade. Numerical MHD simulations (designed for 

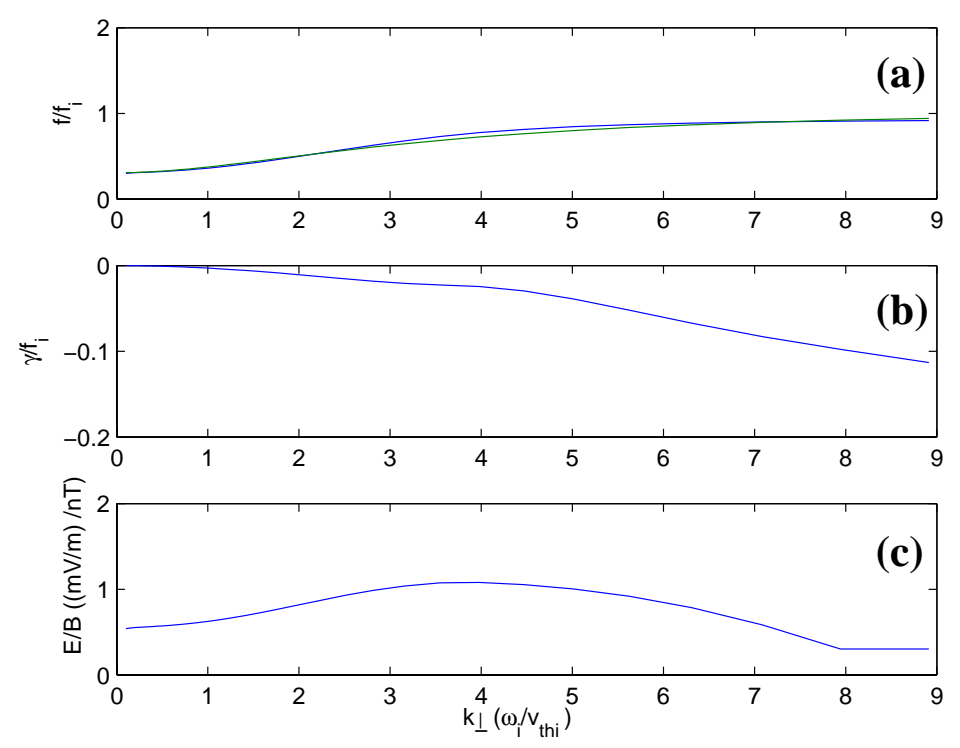

Fig. 8. Solutions from WHAMP code. (a) shows the real frequency of a kinetic Alfvèn wave with $k_{\|}=0.1$ normalized to ion cyclotron frequency at $B=85 \mathrm{nT}$ as a function of $k_{\perp}$ (blue). The green line in (a) shows the solution of the real frequency calculated from the dispersion relation of kinetic Alfvén (Eq. 2) waves normalized to WHAMP units. (b) Damping (imaginary frequency) as a function of $k_{\perp}$. (c) $E_{\perp} / B_{\perp}$-ratio.

open solar coronal magnetic field region) by Dmitruk et al. (2004) show the coexistence of discrete modes and turbulence in a presence of a strong background magnetic field. It therefore may be possible that both energy cascade and linear modes coexist in the cusp geometry (with magnetic field of 100-60 nT and ion beta of 0.02-0.4).

Here we first consider the possibility that the break point frequency may arise due to fluctuations that cannot be resupplied by the cascade because they damp too efficiently. In a collisionless plasma there is no dissipation due to collisions, but dissipation arises due to wave-particle interactions such as Landau and cyclotron damping. Nykyri et al. $(2003,2004)$ show that the waves were propagating at variety of angles with respect to background magnetic field $\left(\theta_{k B}=0^{\circ}-90^{\circ}\right)$, thus all of parallel, obliquely and perpendicularly propagating waves need to be damped to give a spectral break point. To investigate wave damping in the vicinity of the ion cyclotron frequency, we have used the WHAMP (Rönnmark, 1982) code with Maxwellian distributions with number density of $n=10 / \mathrm{cm}^{3}$ and temperatures of $17 \mathrm{eV}(0.2 \mathrm{MK})$ and $170 \mathrm{eV}(2.0 \mathrm{MK})$ for electrons and protons, respectively. These values correspond to measured values by Cluster PEACE and CIS instruments. We run the model with magnetic fields of $85 \mathrm{nT}$ and $60 \mathrm{nT}$, corresponding to different regions in the cusp. There are four wave modes at the vicinity of cyclotron frequency that may be present in the observed spectra: 1) Kinetic Alfvén waves, 2) Ion cyclotron/Alfvén waves, 3) Ion Bernstein waves and 4) Fast/magnetosonic waves. In the following we will discuss their importance in determining the breakpoint frequency.
We require that the imaginary frequency exceeds $20 \%$ of the real frequency in order to ensure that the particular mode is important contributing to the break frequency. We identify the range of parallel $\left(k_{\|}\right)$and perpendicular $\left(k_{\perp}\right)$ wave numbers and $\omega$ for these damped waves and evaluate the Doppler shift for these solutions in the end of this section.

\subsection{Damping of the kinetic Alfvén waves}

Kinetic Alfvén waves (KAW) can be viewed as a coupling of the ion acoustic and shear Alfvén modes (Lysak and Lotko, 1996). They can exist in a plasma with $m_{e} / m_{i} \leq \beta_{e}<1$. For this cusp crossing, the ion beta between 05:00-06:00 UT varies between 0.02 and 0.4 , and the electron and ion temperature ratio, $T_{e} / T_{i}$ is about 0.1 (this can be determined from PEACE data), so that $\beta_{e}$ lies between 0.002 and 0.04 and is within the required range.

In the limit of $\omega<\omega_{i}$, Stasiewicz et al. (2000) derived a dispersion relation for KAW including the finite Larmor radius and finite frequency effects:

$\omega=k_{\|} v_{A} \sqrt{1+k_{\perp}^{2}\left(r_{i}^{2}+r_{s}^{2}\right)-\frac{\omega^{2}}{\omega_{i}^{2}}\left(1+k_{\perp}^{2} r_{i}^{2}\right)}$,

where $r_{i}$ is ion cyclotron radius, $\omega_{i}$ is the ion cyclotron frequency, $v_{A}$ is Alfvén speed, and $r_{s}=\sqrt{k_{b} T_{e} /\left(m_{i} \omega_{i}^{2}\right)}$.

Figure $8 \mathrm{a}$ shows the real frequency of the KAW normalized to the ion cyclotron frequency (for $B=85 \mathrm{nT}$ ) calculated from WHAMP (blue) and from Eq. (2) (green). Although, there is some discrepancy between $k_{\perp}=3-6$, the solution 

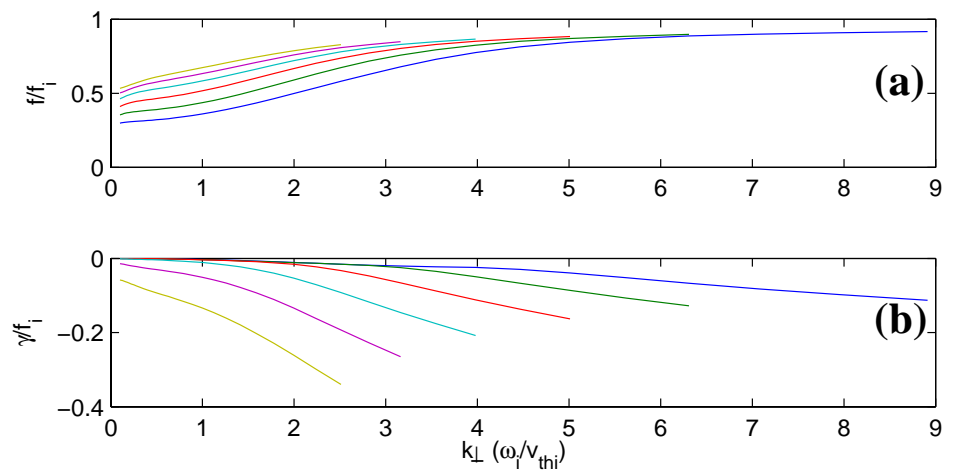

Fig. 9. Solutions from WHAMP code for kinetic Alfvén waves. (a) shows the real part and (b) shows the imaginary part of frequency with $k_{\|}=0.1$ (blue), $k_{\|}=0.13$ (green), $k_{\|}=0.16$ (orange), $k_{\|}=0.2$ (aqua), $k_{\|}=0.25$ (magenta), $k_{\|}=0.32$ (yellow) normalized to ion cyclotron frequency at $B=85 \mathrm{nT}$ as a function of $k_{\perp}$.

Table 2. Properties of Kinetic Alfvén waves (KAW) when damping becomes $20 \%$ of the real frequency (calculated from WHAMP model with Maxwellian electron and proton species with $B=85 \mathrm{nT}$ ). Columns list parallel and perpendicular wave numbers and wave lengths, real frequency and wave propagation angle with respect to magnetic field. Ion cyclotron radius, $r_{t h i}$, used here is $22 \mathrm{~km}$.

\begin{tabular}{lllllll}
\hline KAW & $\mathrm{k}_{\|}\left(1 / r_{t h i}\right)$ & $\mathrm{k}_{\perp}\left(1 / r_{t h i}\right)$ & $\lambda_{\|}(\mathrm{km})$ & $\lambda_{\perp}(\mathrm{km})$ & $\omega / \omega_{i}$ & $\theta_{k B}$ (degrees) \\
\hline 0.32 & 1.0 & 432 & 138 & 0.67 & 72 \\
0.25 & 2.2 & 553 & 63 & 0.78 & 84 \\
0.2 & 3.5 & 691 & 39 & 0.85 & 87 \\
\hline
\end{tabular}

from the dispersion relation matches the WHAMP solution quite nicely with $k_{\|}=0.1$. The wave numbers $k_{\perp}$ and $k_{\|}$are normalized to the inverse gyroradius, $1 / r_{i}=\omega_{i} / v_{t h i}$. Figure $8 \mathrm{~b}$ shows the imaginary part (damping) of the frequency $\left(\gamma / f_{i}\right)$. One can see that damping increases rapidly above $k_{\perp}=4$ and reaches over $10 \%$ of the real frequency at $k_{\perp}=7.9$. The $E_{\perp} / B_{\perp}$-ratio (shown in Fig. 8c) is of the order of unity (in units $(\mathrm{mV} / \mathrm{m}) / \mathrm{nT}$ ). This is of the order of the Alfvén speed indicating that the wave is mostly electromagnetic.

Figure 9a is in the same format as Fig. 8a, but in addition to solution with $k_{\|}=0.1$ (blue), WHAMP solutions with $k_{\|}=0.13$ (green), $k_{\|}=0.16$ (orange), $k_{\|}=0.2$ (aqua), $k_{\|}=0.25$ (magenta) and $k_{\|}=0.32$ (yellow) are shown. Panel (b) shows that damping becomes more important as $k_{\|}$increases.

Let us consider the following physical argument that determines the values of $k_{\perp}$ at which dissipation of KAW becomes significant. Hasegawa and Chen (1976) state that in the collisionless regime if $\beta>(<) 0.1$, ion (electron) Landau damping dominates the dissipation of the KAW. Here the ion beta varies between $0.02-0.4$ so it is likely that either damping mechanism may occur in different regions of the cusp. Let us first consider electron Landau damping. By neglecting the finite frequency effects in Eq. (2), it can be shown that when $\omega / k_{\|}=V_{A} \sqrt{1+\left(1+T_{e} / T_{i}\right) k_{\perp}^{2} r_{i}^{2}} \approx V_{\text {the }}$, (where the electron thermal speed, $V_{\text {the }}=\sqrt{\frac{\beta_{i} T_{e} m_{i}}{2 T_{i} m_{e}}} V_{A}$ ), the dissipation becomes significant for $k_{\perp} \approx(1-6) / r_{i}$ (corresponding to $\left.\beta_{i}=0.02-0.4\right)$. The maximum heating rate for ions due to the ion Landau damping is obtained with $k_{\perp} r_{i}=1$ (Hasegawa and Chen, 1976).

Table 2 lists the properties of the KAW when damping becomes $20 \%$ of the real frequency. Only solutions with $k_{\|}>0.19$ have damping rates exceeding $20 \%$ of the real frequency. Calculations of the Doppler shifted theoretical spectral breakpoint frequencies of the KAW waves in Fig. 13c (Fig. 13 will be fully discussed at the end of this section) use these values for $k_{\|}, k_{\perp}, \omega$, and $\theta_{k B}$. It can be seen that damping of the obliquely propagating $\left(\theta_{k B}=72-87^{\circ}\right) \mathrm{KAW}$ can lead to the observed spectral breakpoint frequency. This implies parallel wave lengths of $430-690 \mathrm{~km}$ and perpendicular wave lengths of $40-140 \mathrm{~km}$ (the ion gyroradius used here is $22 \mathrm{~km}$ (for $T_{i}=2 \mathrm{MK}, B=85 \mathrm{nT}$ )). Table 3 is in the same format as Table 2 showing WHAMP solutions for $B=60 \mathrm{nT}$ $\left(r_{i}=31 \mathrm{~km}\right)$. These solutions are very similar to those of Table 2 .

\subsection{Damping of the Alfvén/ion cyclotron modes}

Figure 10 is in the same format as Fig. 8a and shows the real and Fig. 8b imaginary frequencies, and Fig. $8 \mathrm{c}$ the $E_{\perp} / B_{\perp}$-ratio of ion cyclotron (IC) waves as a function of $k_{\|}$with $k_{\perp}=0$. The damping rate increases significantly above $k_{\|}=0.4$ and becomes $20 \%$ of the real frequency 
Table 3. Properties of Kinetic Alfvén waves (KAW) when damping becomes $20 \%$ of the real frequency (calculated from WHAMP model with Maxwellian electron and proton species with $B=60 \mathrm{nT}$ ). Columns list parallel and perpendicular wave numbers and wave lengths, real frequency and wave propagation angle with respect to magnetic field. Ion cyclotron radius, $r_{\text {thi }}$, used here is $31 \mathrm{~km}$.

\begin{tabular}{lllllll}
\hline KAW & $\mathrm{k}_{\|}\left(1 / r_{\text {thi }}\right)$ & $\mathrm{k}_{\perp}\left(1 / r_{\text {thi }}\right)$ & $\lambda_{\|}(\mathrm{km})$ & $\lambda_{\perp}(\mathrm{km})$ & $\omega / \omega_{i}$ & $\theta_{k B}$ (degrees) \\
\hline 0.32 & 2.0 & 612 & 98 & 0.71 & 81 \\
0.25 & 3.2 & 783 & 61 & 0.8 & 85 \\
0.2 & 5.0 & 980 & 39 & 0.86 & 88 \\
0.16 & 7.2 & 1224 & 27 & 0.86 & 89 \\
\hline
\end{tabular}
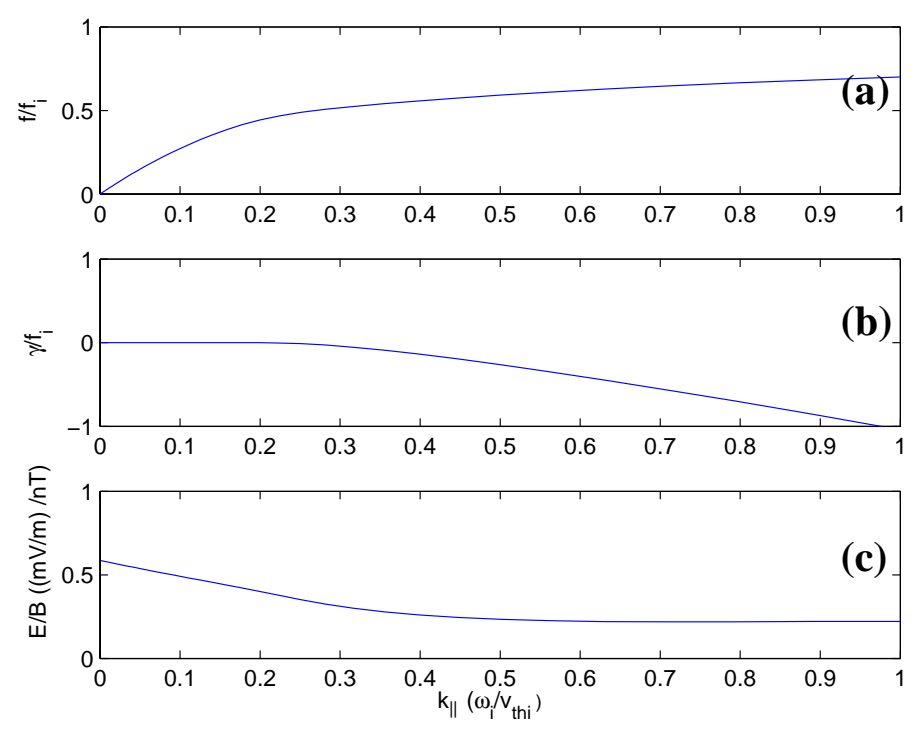

Fig. 10. Solutions from WHAMP code. (a) shows the real part, (b) shows the imaginary part of frequency normalized to $f_{I C}$ at $B=85 \mathrm{nT}$ and (c) shows the $E_{\perp} / B_{\perp}$-ratio of the ion cyclotron wave as a function of $k_{\|}$with $k_{\perp}=0$.

at $\approx 0.55 f_{I C}$. The wave is mostly magnetic with the $E_{\perp} / B_{\perp}$-ratio being of the order of the local Alfvén speed (in units $(\mathrm{mV} / \mathrm{m}) / \mathrm{nT})<1$ ). Figure 11 is in the same format as Fig. 10a and 10b, but in addition solutions with $k_{\perp}=0.79$ (orange), $k_{\perp}=1.0$ (aqua), $k_{\perp}=1.26$ (magenta), $k_{\perp}=1.58$ (yellow), $k_{\perp}=2.0$ (gray), $k_{\perp}=2.51$ (blue) and $k_{\perp}=3.16$ are shown. Table 4 shows properties of these waves when damping becomes $20 \%$ of the real frequency. It can be seen that ion cyclotron modes with all angles of propagation $\left(\theta_{k B}=0-86^{\circ}\right)$ are efficiently damped. This implies parallel wave lengths of $360-660 \mathrm{~km}$ and minimum perpendicular wave lengths of $40 \mathrm{~km}$ (the ion thermal gyroradius used here is $22 \mathrm{~km}$ (for $T_{i}=2 \mathrm{MK}, B=85 \mathrm{nT}$ )). Calculation of the Doppler shifted theoretical spectral breakpoint frequency of the IC waves in Fig. 13d uses the solutions listed in Table 4. WHAMP calculations with $B=60 \mathrm{nT}$ (not shown) indicate that the damping of the parallel propagating solution reaches $20 \%$ at $k_{\|}=0.42$ and $\omega=0.46 \omega_{i}$, which is at lower frequency than the corresponding solution calculated with $B=85 \mathrm{nT}$. Also the obliquely propagating solutions reach the $20 \%$ damping at lower frequencies for $B=60 \mathrm{nT}$.

\subsection{Damping of the Ion Bernstein waves}

Figure 12 is in the same format as Fig. 8 showing the real (a) and imaginary (b) frequencies, and the $E_{\perp} / B_{\perp}$-ratio (in units $(\mathrm{mV} / \mathrm{m}) / \mathrm{nT}$ ) (c) of the ion Bernstein (IB) waves as a function of $k_{\perp}$. Several curves (with increments of 0.003 and with cyclic color table) are shown with $k_{\|}=0.01-0.06$. When $k_{\perp}<0.7$, the real frequency increases to $1.8 f_{I C}$ for all the solutions. When $k_{\perp}$ increases the real frequency gradually decreases toward $f_{I C}$, but then diverges again for solutions with highest $k_{\|}$.

Maximum damping is obtained for solution with $k_{\|}=0.06$ (orange) (b), but remains still less than $5 \%$ of the real frequency below $k_{\perp}=10$. For the most damped solution $\mathrm{f}=1.4 f_{I C}, k_{\|}=0.06, k_{\perp}=10$, and $\theta_{k B}=89.7^{\circ}$

Damping is negligible for solutions with $k_{\|}<0.02$. For increased $k_{\|}$the waves have a magnetic component but become more electrostatic as $k_{\|}$decreases (c): for $k_{\|}=0.01$, the $E_{\perp} / B_{\perp}$-ratio is 30 times larger than for $k_{\|}=0.06$. When $k_{\|}=0$, the wave is entirely electrostatic (not shown). Based on these WHAMP calculations, damping of the IB modes is not 

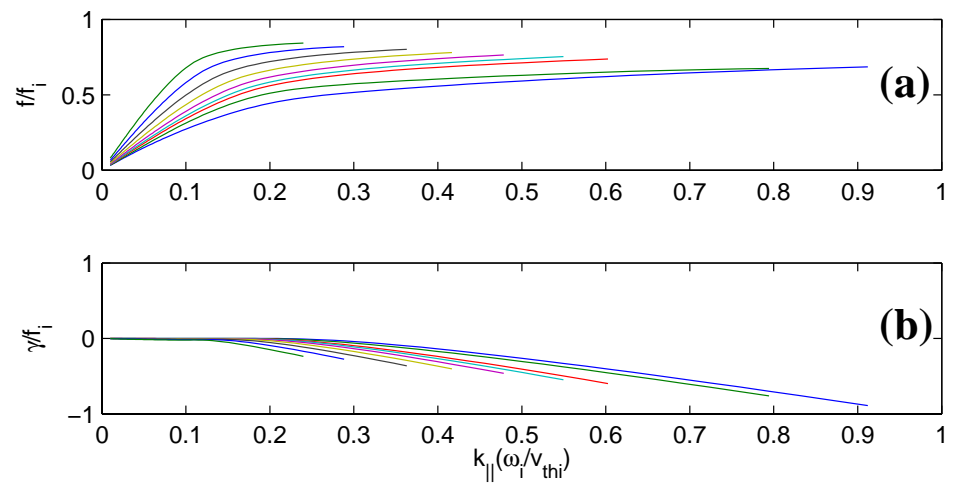

Fig. 11. Solutions from WHAMP code. (a) shows the real part and (b) shows the imaginary part of frequency of the ion cyclotron wave with $k_{\perp}=0$ (blue solution continuing to highest $k_{\|}$), $k_{\perp}=0.32$ (green), $k_{\perp}=0.79$ (orange), $k_{\perp}=1.0$ (aqua), $k_{\perp}=1.26$ (magenta), $k_{\perp}=1.58$ (yellow), $k_{\perp}=2.0$ (gray), $k_{\perp}=2.51$ (blue), $k_{\perp}=3.16$ (green) normalized to ion cyclotron frequency at $B=85 \mathrm{nT}$ as a function of $k_{\|}$.

Table 4. Properties of Ion Cyclotron waves (IC) when damping becomes $20 \%$ of the real frequency (calculated from WHAMP model with Maxwellian electron and proton species). Columns list parallel and perpendicular wave numbers and wave lengths, real frequency and wave propagation angle with respect to magnetic field. Ion cyclotron radius, $r_{t h i}$, used here is $22 \mathrm{~km}$.

\begin{tabular}{lllllll}
\hline IC & $\mathrm{k}_{\|}\left(1 / r_{\text {thi }}\right)$ & $\mathrm{k}_{\perp}\left(1 / r_{\text {thi }}\right)$ & $\lambda_{\|}(\mathrm{km})$ & $\lambda_{\perp}(\mathrm{km})$ & $\omega / \omega_{i}$ & $\theta_{k B}$ (degrees) \\
\hline 0.38 & 0.0 & 364 & $\mathrm{Inf}$ & 0.55 & 0 \\
0.36 & 0.1 & 395 & 1382 & 0.56 & 16 \\
0.35 & 0.32 & 395 & 432 & 0.59 & 42 \\
0.34 & 0.79 & 407 & 175 & 0.66 & 67 \\
0.32 & 1.0 & 432 & 138 & 0.67 & 72 \\
0.31 & 1.26 & 446 & 110 & 0.70 & 76 \\
0.29 & 1.58 & 477 & 87 & 0.73 & 80 \\
0.26 & 2.0 & 531 & 69 & 0.76 & 83 \\
0.24 & 2.51 & 576 & 55 & 0.80 & 85 \\
0.21 & 3.16 & 658 & 44 & 0.83 & 86 \\
\hline
\end{tabular}

as efficient as damping of the KAW or IC waves for the prevailing cusp conditions. The IB mode is coupled to the magnetosonic mode at $k_{\perp}<0.5$ and $f<1.8 f_{I C}$ (not shown here), but the damping for that mode is negligible below $k_{\perp}<1$ and at the vicinity of the $f_{I C}$.

\subsection{Discussion on Doppler effect and summary}

Figure 13 summarizes the results of this section. The first panel shows the observed slopes at the inertial range every minute (using a 8192 pt. window). The second panel shows the ratio of the observed spectral break point frequency $\left(f_{b f}\right)$ to the local ion cyclotron frequency for all four spacecraft between 05:00-06:00 UT (for the same spectra as calculated for panel a). The empty points indicate that the break point was not clearly identifiable. The observed spectral breakpoint frequency is at the vicinity of the local cyclotron frequency between 05:08-05:28 UT, and usually below $f_{I C}$ after 05:30 UT. There are few points where the observed break is above the $f_{I C}$.
As discussed above, only the KAW and IC waves are efficiently damped, so we will calculate the Doppler shift (according to Eq. 1) for each of these wave modes using the values of $k_{\perp}, k_{\|}, \omega$ and $\theta_{k B}$ listed in Tables 2 and 4. The Doppler shift is calculated for every minute and the plasma parameters used in these calculations are averaged using a 11.4-s window and $\theta_{k V}=\theta_{B V}-\theta_{k B}$. We note that the spectral calculations are using 6.1-min window, so that such a spectra has a superposition of the different Doppler-shifted wave modes. However, using 6.1-min averaging of the plasma parameters will underestimate the Doppler-shift for some intervals, which is why we use the higher resolution in order to get a broader range of Doppler-shifts. In addition to the waves with $\theta_{k B}$, the Doppler shift is also calculated for $\theta_{k B}+180^{\circ}$, corresponding to the solutions where $\mathbf{k}$ has a component anti-parallel to the magnetic field (i.e. waves propagating tailwards). Panels (c)-(d) show the ratio of the Doppler shifted theoretical spectral breakpoint frequency to $f_{I C}$ for $B=85 \mathrm{nT}$ (corresponding to time at $\approx 05: 15 \mathrm{UT}$ ) for KAW and IC waves, respectively. The panels (c) and (d) 

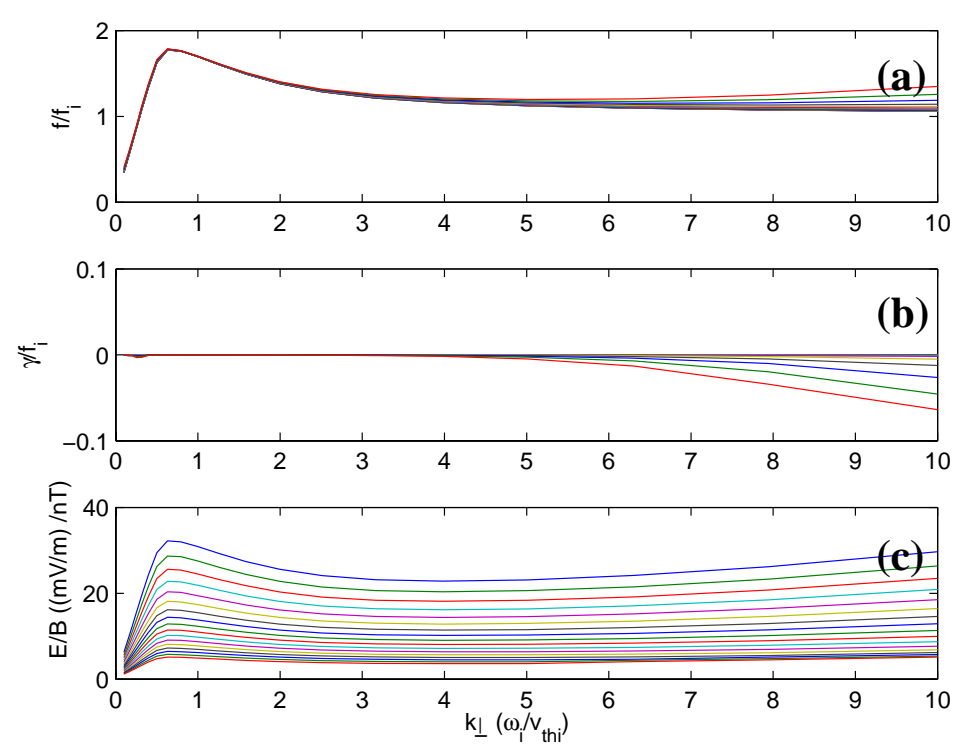

Fig. 12. Solutions from WHAMP code. (a) shows the real part and (b) shows the imaginary part of frequency of ion Bernstein modes with $k_{\|}=0.06$ (orange) $-k_{\|}=0.01$ (blue among cluster of lines) normalized to ion cyclotron frequency at $B=85 \mathrm{nT}$ as a function of $k_{\perp}$. (c) shows $E_{\perp} / B_{\perp}$-ratios. Note that a cyclic color table is used such that $k_{\|}=0.06\left(k_{\|}=0.01\right)$ is corresponding to the curve with lowest (highest) $E_{\perp} / B_{\perp}$-ratio and that $k_{\|}=0.06$-solution is most strongly damped (b).

are assuming that the magnetic field magnitude is at constant $85 \mathrm{nT}$, although the field gradually decreases and has slightly different magnitudes at all spacecraft. The WHAMP curves look quite similar at $B=60 \mathrm{nT}$ (corresponding to time at $\approx 05: 40 \mathrm{UT}$ ) for KAW, and show damping at lower frequencies for IC waves.

During times of enhanced flows (associated with D-shaped distributions) the Doppler shift generates a larger envelope in frequency space between parallel and anti-parallel propagating solutions (Figs. 13c and d): the waves that are generated below (above) the spacecraft and that are propagating against (along) the flow have decreased (increased) frequency from the plasma frame. Sundkvist et al. (2005) show that D-shaped distributions could generate waves along the field lines (propagating in both directions) at the vicinity of the local ion cyclotron frequency. Between 05:08-05:10 UT sc4 is observing the break frequency, $f_{b f}$, below $0.4 f_{I C}$ in agreement with the Doppler-shifted solutions of the waves that propagate against the flow (reduced frequency from the plasma frame). During this interval the other three spacecraft observe the break between $0.7-0.9 f_{I C}$. These correspond quite nicely to the IC modes that have a small $\theta_{k B}$ and propagate along the flow (increased frequency from the plasma frame). Between 05:10-05:12 UT the Doppler-shifted solutions do not match the observed break frequencies. The observed $f_{b f}$ are in fact closer to theoretical frequencies in the plasma frame (see column 6 in Tables 3 and 4). The absolute magnitudes for the Doppler-shift during this interval are in range of $0.3-2 f_{I C}$, so that the waves causing the break point frequency at $0.7-0.9 f_{I C}$ could be formed by a) con- vecting spatial structures (zero plasma frequency) along the flow, b) propagating waves that are generated in low field region along the flow, or c) waves that are generated in the high field region and are propagating against the flow. Between 05:12-05:27 UT there is a range of Doppler-shifted waves that match the observed $f_{b f}$. Between 05:30-05:34 UT the Doppler-shift is small, and yet the observed break points differ from the theoretical break points in the plasma frame. This could indicate that the fluctuations causing the break point are generated in the lower field region and propagate to Cluster. Between 05:35-05:42 UT the break point can be explained by the Doppler shifted obliquely propagating waves that have a component of the $k$-vector against the flow. Between 05:47-05:48 UT sc1 and sc3 measure break points that can be explained by damping of the waves that propagate with oblique angles and have $k$-vectors along the flow, but sc 4 observes the break at much lower frequencies and not in agreement with the calculated Doppler-shifted solutions at $85 \mathrm{nT}$, which could indicate that these fluctuations are generated in the low field region and propagating along the flow (20\% damping of the IC modes occurs at lower frequencies at low field region). The same is true also for all intervals after 05:50 UT at sc4 and sc3, and for two intervals at sc1. In summary the observed break frequencies could be explained by the wave damping of KAW and IC waves assuming these waves are generated both locally as well as below and above the spacecraft trajectory. We have assumed here that the imaginary frequency must become $20 \%$ of the real frequency in order to ensure that the particular wave mode is contributing to the break point. If lower values e.g. $10 \%$ 


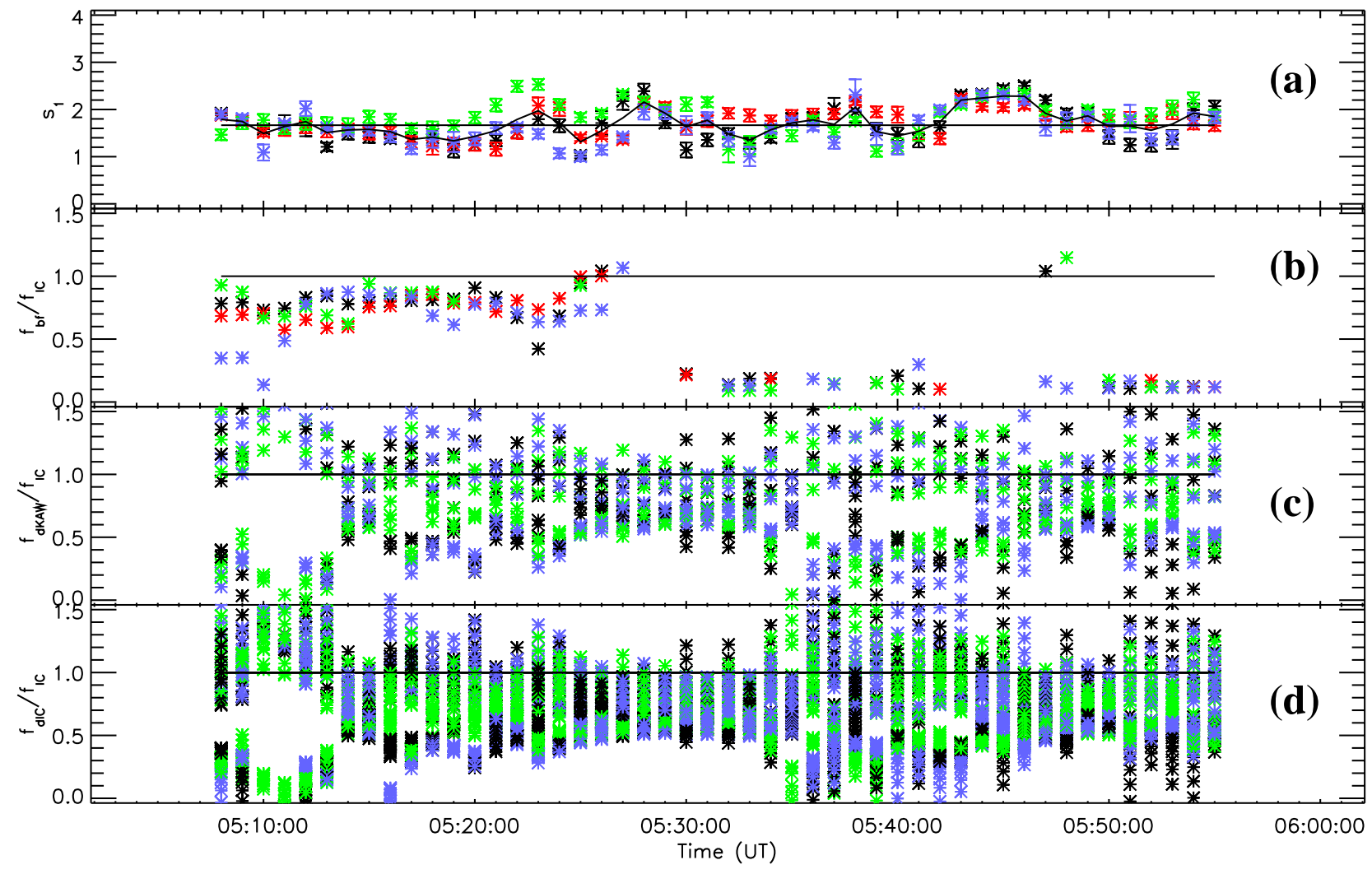

Fig. 13. (a) The inertial range slopes calculated every minute using a 8192-point window with FGM data. (b) The ratio of the observed spectral breakpoint frequency $f_{b f}$ to $f_{I C}$ for the same spectra as calculated for panel (a). The empty points indicate that the break point was not clearly identifiable. Panels (c)-(d) show the ratio of the Doppler shifted theoretical spectral breakpoint frequency to $f_{I C}$ (at $B=85 \mathrm{nT}$ ): (c) shows the kinetic Alfvén wave solutions with values of $k_{\perp}, k_{\|}, \theta_{k B}$ listed in Table 2 (d) shows the IC wave solutions according to Table 4. Note that the Doppler shift for each solution is calculated both for $\theta_{k B}$ and $\theta_{k B}+180^{\circ}$. The colors correspond to different spacecraft and the Doppler shift is not calculated for sc2 due to lack of plasma data. The calculation of the Doppler-shift uses 11.4-s averages of variables $V$ and $\theta_{k V}$ in Eq. (1).

are used, the plasma frame frequency used in Doppler shift calculations would move to lower frequencies and $\theta_{k B}$ would slightly decrease for KAW (see Figs. 9 and 11).

During times when cyclotron harmonics are observed at the stagnant cusp boundary (between 05:25-05:29 UT and 05:45-05:49 UT, see also Figs. 10 and 12 of Nykyri et al., 2004) the Doppler effect is small and these wave trains are more clearly structured and last longer than the waves observed during the sheared flows. It thus appears that the wave modes at the stagnant cusp boundary are observed because either a) they are not efficiently damped, or b) because the spacecraft are passing through the region where they are constantly being generated, rather than being convected by the flow.

We have here considered the possibility that the broadband power law spectra may be composed of an inertial range and a dissipation range. The inertial range fluctuations could be generated by lobe reconnection and associated shear flow layers. An energy cascade may take place perpendicular to the magnetic field (approximately along Cluster trajectory).
The break point frequency could be generated by efficient damping of obliquely propagating KAW modes and ion cyclotron modes.

Another possibility (private communication from D. Sundkvist) is that instead of single inertial and dissipation ranges, the spectra is formed of two inertial ranges. It may be possible that the first inertial range is caused (as described above) by the cascade of the fluctuations driven by the lobe reconnection time scale. The second inertial range could be caused by the cascade of the fluctuations generated by several sources at the vicinity of the local ion cyclotron frequency.

We also cannot rule out the possibility that the broadband nature of the spectra is formed partly by the Doppler shift of the different wave modes. However, the broad band nature of the spectra (with a single slope) is also present between 05:28-05:34 UT, when there is no plasma flow and thus no Doppler-effect. For this interval the slopes at the inertial range are $-1.35,-1.75,-2.26$ and -1.83 , for $\mathrm{sc} 1$, sc2, $\mathrm{sc} 3$, and sc4, respectively. 

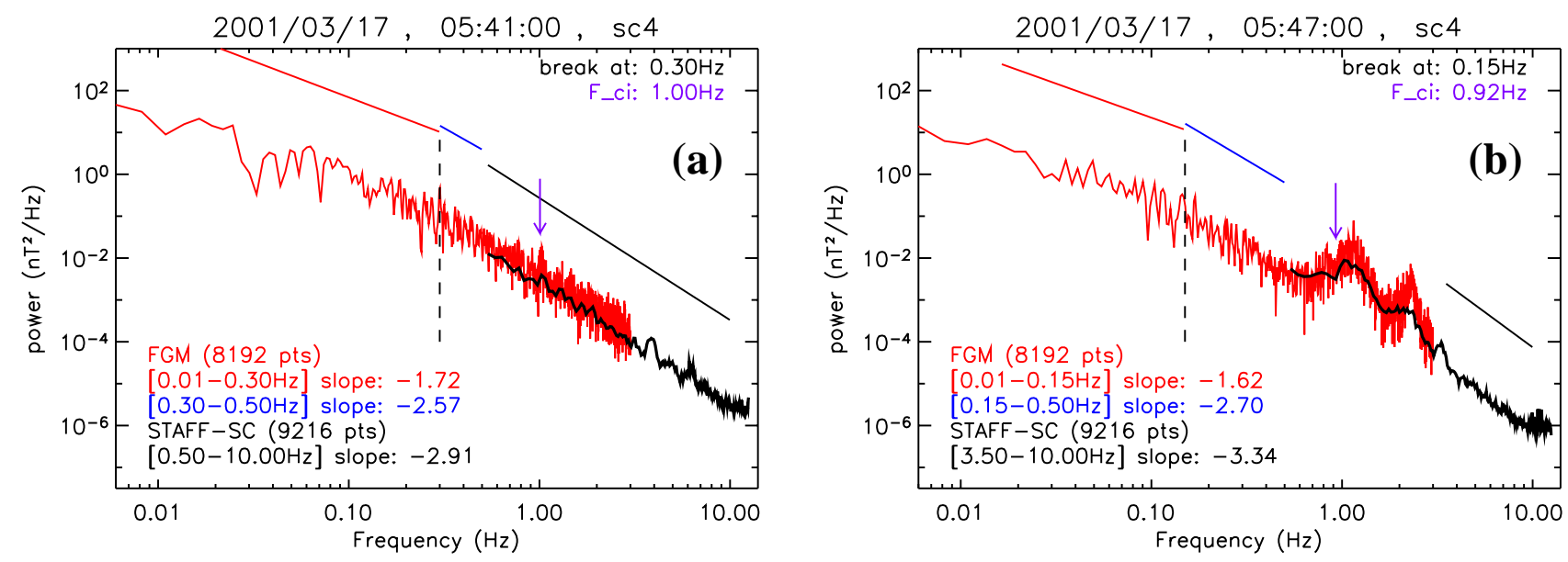

Fig. 14. Power spectra measured by sc4 at 05:41 UT (a) and at 05:47 UT (b) and their spectral slopes at inertial and dissipation range.

Smaller spacecraft separations (of the order of $r_{i}$ ) would be required in order to accurately determine the Doppler effect for the each wave mode at the vicinity of the ion cyclotron frequency, by using the $k$-filtering technique. Even better would be to have a cluster of clusters covering different scales, from MHD to kinetic ion and electron scales.

\section{Feeding of the dissipation range}

We suggested earlier that the inertial range fluctuations that are in the frequency range of FGM measurements are generated both remotely (perhaps due to lobe reconnection) and locally due to sheared flows arising from reconnection process. In addition, there are sometimes occasions when local processes generate the harmonic peaks, and these peaks can then feed the dissipation range. Some evidence for this is shown in Fig. 14 which presents power spectral slopes measured by sc4 during 6.1-min intervals centered at 05:41 UT (left) and 05:47 UT (right).

The shape of the spectra during these two intervals is very different: the 05:41 UT-centered interval shows a more continuous spectra with a not-so-clearly observable break point, whereas the 05:47 UT-centered interval shows a clearer break at $0.14 \mathrm{~Hz}$ with harmonics in the dissipation range.

Slopes in the inertial range are -1.72 and -1.62 (calculated from the FGM between $0.015-0.3 \mathrm{~Hz}$ (a) and between 0.015 $0.14 \mathrm{~Hz}(\mathrm{~b})$ ), respectively. The break frequency is determined visually and its location can be confirmed by the steepened slope (measured by FGM) of -2.57 between $0.3-0.5 \mathrm{~Hz}$ (a) and -2.7 between $0.15-0.5 \mathrm{~Hz}$ (b). The purple arrow marks the local ion cyclotron frequency, so for both intervals the break is observed below the proton cyclotron frequency. The dissipation range slope at $05: 41$ UT is -2.91 and is calculated from STAFF between $0.5-10.0 \mathrm{~Hz}$. In the 05:47 UTcentered interval there is also a steeper slope of -3.34 mea- sured from STAFF between $3.5-10 \mathrm{~Hz}$ (which is above the spectral peaks).

The interval centered at 05:41 UT includes an interval of parallel flow of $\approx 100 \mathrm{~km} / \mathrm{s}$, presumably associated with lobe reconnection. The total integrated power in the inertial range calculated from FGM between $0.015-0.3 \mathrm{~Hz}$ is $0.39 \mathrm{nT}^{2}$, and the dissipation range power calculated from STAFF between $0.6-10 \mathrm{~Hz}$ is $0.0035 \mathrm{nT}^{2}$.

The interval centered at 05:47 UT is during a time when coherent wave trains (similar to 05:26-05:29 UT) with harmonics of the ion cyclotron frequency were observed (see also Figs. 12 and 13 in Nykyri et al. (2004)). The total integrated power between $0.015-0.14 \mathrm{~Hz}$ (from FGM) at this time is $0.098 \mathrm{nT}^{2}$, which is a factor four smaller than during the 05:41 UT-centered interval. However, the fundamental harmonic peak (slightly above $f_{I C}$ ), and its first harmonic, have piled up power in the dissipation range. The integrated power measured by STAFF between $0.6-10 \mathrm{~Hz}$ is $0.0047 \mathrm{nT}^{2}$ which is about 1.3 times larger than during the 05:41 UTcentered interval. This seems to indicate that some of the power from the inertial range has shifted into the dissipation range. However, we note here that this is not just a temporal effect but spatio-temporal because the spacecraft have moved $\sim 2300 \mathrm{~km}$ between 05:37 UT and 05:50 UT (from the beginning of the measurements used in 05:41 UT-centered interval to end of measurements used in 05:47 UT-centered interval).

\section{$7 \quad$ Discussion and conclusions}

We have used combined data from the Cluster STAFF and FGM instruments to evaluate slopes of the magnetic field power spectra in the high-altitude cusp. We found examples of double sloped, broadband spectra with shallower "inertial" and steeper "dissipation" range: the inertial range slopes vary between -2.7 to -1 . The dissipation range slopes vary 
between -5 to -3 . For most of the intervals the spectral breakpoint was clear and occurred at the vicinity of the local ion cyclotron frequency $\left(f_{I C}\right)$. We also found examples of single sloped spectras where break was not as clearly identifiable (see Fig. 14a). During some intervals clear peaks at cyclotron harmonics were superimposed on this broad band spectra

Slopes of the power spectra both in the inertial and dissipation ranges vary throughout the cusp crossing, and even in the same intervals can differ by $100 \%$ at different spacecraft. Although the plasma properties vary on scales of the spacecraft separation $(\sim 600 \mathrm{~km})$, we found no clear correlation between any local plasma parameters and the power spectra slopes. However, we did find a correlation between the integrated power of the magnetic field fluctuations and plasma number flux (this is also observed in recent studies by Sundkvist et al., 2005; Grison et al., 2005), indicative that the solar wind interaction with the cusp magnetic field due to lobe reconnection provides free energy for these fluctuations to grow. We also calculated the ratio between the perpendicular and parallel power indicating that in both ranges the fluctuations are mostly transverse.

It may be possible that the cusp geometry with reasonably strong magnetic field (100-60 nT) allows the coexistence of both turbulence and discrete wave modes. Indeed, this is shown to be possible in simulations corresponding to the open coronal magnetic field lines (Dmitruk et al., 2004). We have considered two mechanisms for the generation of the spectral breakpoint and double sloped spectra:

1. In terms of inertial and dissipation range: The onset of the inertial range fluctuations $\left(<f_{I C}\right)$ could be formed partly by the Alfvénic fluctuations that are generated in the lobe reconnection process (propagating Earthwards) and partly by the velocity shears that arise from the reconnection process. Indeed, we have observed velocity shears of $\sim 100 \mathrm{~km} / \mathrm{s}$ at the spacecraft separation scale of $600 \mathrm{~km}$. The broadband (power law) nature of the spectra may be formed due to the turbulent cascade (operating perpendicularly to the ambient magnetic field) of these fluctuations. The onset of the dissipation range may be caused by damping of the fluctuations that cannot be re-supplied by the cascade from the inertial range. We have used the WHAMP code to study the linear damping properties of the waves at the vicinity of the local ion cyclotron frequency. The results show that an efficient damping $(20 \%$ of the real frequency) is occuring for obliquely propagating kinetic Alfvén modes with $\theta_{k B}=70-90^{\circ}$, and for ion cyclotron modes with all angles of propagation. Damping of the magnetosonic modes and ion Bernstein modes is negligible.

2. In terms of two inertial ranges: The first inertial range is generated as described above (one source being at the
MHD frequencies due to lobe reconnection). The second inertial range may be formed by cascading of the fluctuations that are generated by one or several sources at the vicinity of the local cyclotron frequency. The observations of the single slope spectra at 05:41 UT could indicate that these sources were absent at the vicinity of the $f_{I C}$, and the spectra is formed by a single inertial range during this interval. Alternatively, (supporting the first mechanism) it may be possible that during this interval the energy cascading time scale is shorter than the damping time scale of the waves.

We will study this possibility of the second inertial range in future by studying unstable distribution functions. Because cusp is not homogeneous, dispersion solvers including gradients in the plasma flow would also be necessary.

In order to understand the formation of the first inertial range we plan to do comparisons between the observed inertial range slopes with high-resolution 3-D MHD cusp simulations. Cuts can be taken through the simulation box in agreement with the real Cluster trajectory. Initial results (Adamson et al., 2005) of this comparison look highly promising, suggesting that lobe reconnection process and resulting velocity shears play role in generating inertial range fluctuations in the high-altitude cusp.

Another interesting topic is the generation of the ion cyclotron harmonics. They occur at the stagnant cusp boundary (05:25-05:29 UT and 05:45-05:49 UT), are electromagnetic in nature and have quite symmetric distribution functions. Ion Bernstein waves are mostly electrostatic and are generated by the shell distributions, excluding them as a possible source for the observed harmonics. One possibility is that ion harmonics are generated by the strong velocity shear but have propagated and observed by Cluster at the stagnant cusp boundary. Figures 9 and 10 of Nykyri et al. (2004) show that harmonics appear after 05:25 UT, about 2 min later than the observed large $(\sim 100 \mathrm{~km} / \mathrm{s})$ velocity shear at 05:23 UT. It is well known that field-aligned velocity shears can excite waves at many harmonics of the $\Omega_{i}$ (Gavrishchaka et al., 2000; Ganguli et al., 2002). However, if this is the case it is surprising why harmonics are not observed when the local velocity shear is the strongest. There are smaller scale $\approx 5-40 \mathrm{~km} / \mathrm{s}$ gradients in velocity during the harmonics observations (i.e. at 05:47:30 UT), so perhaps the velocity shear mechanism is able to generate harmonics only in narrow range favoring lower magnitudes for shear.

Acknowledgements. Cluster work in the UK and France is supported by PPARC and CNES respectively. P. J. Cargill also thanks PPARC for the award of a Senior Research Fellowship. We thank J. R. Johnson at Princeton University for helpful discussions on KAW. We also acknowledge D. Sundkvist for suggesting that the double slope spectra could be generated by two inertial ranges. We thank S. Schwartz for confirming the electron temperatures from the PEACE data.

Topical Editor T. Pulkkinen thanks G. Ganguli and another referee for their help in evaluating this paper. 


\section{References}

Adamson, E. T., Otto, A., and Nykyri, K.: Plasma dynamics in the vicinity of a cusp-like magnetic configuration, SM52A-08 AGUfall meeting, 2005.

Balogh, A., Dunlop, M. W., Cowley, S. W. H., Southwood, D. J., Thomlinson, J. G., and magnetometer team: The Cluster Magnetic Field Investigation, Space Sci. Rev., 79, 65-91, 1997.

Balogh, A., Carr, C. M., Acuña, M. H., Dunlop, M. W., Beek, T. J., Brown, P., Fornangon, K.-H., Georgescu, E., Glassmeier, K.-H., Harris, J., Musmann, G., Oddy, T., and Schwingenschuh, K.: The Cluster magnetic field investigation: overview of in-flight perfomance and initial results, Ann. Geophys., 19, 1207-1217, 2001.

Cargill, P. J., Dunlop, M. W., Lavraud, B., Elphic, R. C., Holland, D. L., Nykyri, K., et. al.: CLUSTER encounters with the high altitude cusp: Boundary Structure and magnetic field depletions, Ann. Geophys., 22, 1739-1754, 2004.

Cargill, P. J., Lavraud, B., Owen, C. J., Grison, B., Dunlop, M. W., Cornilleau-Wehrlin, N., Escoubet, C. P., Paschmann, G., Phan, T. D., L.Rezeau, Bogdanova, Y., and Nykyri, K.: The Cusp, Outer Magnetospheric Boundaries:Cluster Results, ISSI, Bern, 2005.

Chen, J. S. and Fritz, T. A.: Correlation of cusp MeV helium with turbulent ULF power spectra and its implications, Geophys. Res. Lett., 25, 4113-4116, 1998.

Cornilleau-Wehrlin, N., Chauveau, P., Louis, S., Meyer, A., Nappa, J. M., Perraut, S., Rezeau, L., Robert, P., Roux, A., Villedary, C. D., Conchy, Y. D., Friel, L., Harve, C. C., Hubert, D., lacombe, C., Manning, R., Wouters, F., Lefeuvre, F., Parrot, M., Pincon, J. L., Poirier, B., Kofman, W., and Louarn, P.: The Cluster Spatio-Temporal analysis of field fluctuations STAFF Experiment, Space Sci. Rev., 79, 107-136, 1997.

Cornilleau-Wehrlin, N., Chanteur, G., Perraut, S., Rezeau, L., Robert, P., Roux, A., de Villedary, C., Canu, P., Maksimovic, M., de Conchy, Y., Lacombe, D. H. C., Lefeuvre, F., Parrot, M., Pinçon, J. L., Décréau, P. M. E., Harvey, C. C., Louarn, P., Santolik, O., Alleyne, H. S. C., Roth, M., Chust, T., Le Contel, O., and Staff Team: First results obtained by the Cluster STAFF experiment, Ann. Geophys., 21, 437-456, 2003.

Cowley, S.: The causes of convection in the Earth's magnetosphere, A review of developments during the IMF, Rev. Geophys, 20, 531-565, 1982.

Dmitruk, P., Matthaeus, W. H., and Lanzerotti, L. J.: Discrete modes and turbulence in a wave-driven strongly magnetized plasma, Geophys. Res. Lett., 31, doi:10.1029/2004GL021119, 2004.

Dunlop, M. W., Cargill, P. J., Stubbs, T. J., and Woolliams, P.: The high altitude cusps: HEOS 2, J. Geophys. Res., 105, 27509 27 517, 2000.

Fuselier, S. A., Trattner, K. J., and Petrinec, S. M.: Cusp observations of high- and low-latitude reconnection for northward interplanetary magnetic field, J. Geophys. Res., 105, 253-266, 2000.

Ganguli, G., Slinker, S., Gavrishchaka, V., and Scales, W.: Low frequency oscillations in a plasma with spatially variable fieldaligned flow, Phys. Plasmas, 9, 2321-2329, 2002.

Gavrishchaka, V., Ganguli, G., Scales, W., Slinker, S., Chaston, C., McFadden, J., Ergun, R., and Carlson, C.: Multiscale coherent structures and broadband waves due to parallel inhomogeneous flows, Phys. Rev. Lett., 85, 4285-4288, 2000.
Goldstein, M. L., Roberts, D. A., and Fitch, C. A.: Properties of the fluctuating magnetic helicity in the inertial and dissipation ranges of the solar wind turbulence, J. Geophys. Res., 99, 11 519-11 538, 1994.

Grison, B., Sahraoui, F., Lavraud, B., Chust, T., CornilleauWehrlin, N., Réme, H., Balogh, A., and Andre, M.: Wave particle interactions in the high-altitude polar cusp: A Cluster case study, Ann. Geophys., 23, 3699-3713, 2005.

Hasegawa, A. and Chen, L.: Kinetic processes in plasma heating by resonant mode conversion of Alfvén wave, Phys. Fluids, 19 (12), 1924-1934, 1976.

Janhunen, P., Olsson, A., Vaivads, A., and Peterson, W. K.: Generation of Bernstein waves by ion shell distributions in the auroral region, Ann. Geophys., 21, 881-891, 2003.

Kessel, R. L.,Chen, S.-H., Green, J. L., Fung, S. F., Boardsen, S. A., Tan, L., Eastman, T. E., Craven, J. D., and Frank, L. A.: Evidence of high-latitude reconnection during northward IMF: Ha wkeye observations, Geophys. Res. Lett., 23, 583-586, 1996.

Kindel, J. M. and Kennel, C. F.: Topside current instabilities, J. Geophys. Res., 76, 3055-3078, 1971.

Kolmogoroff, A. N.: The local structure of turbulence in incompressible viscous fluid for very large Reynolds number, Dokl. Akad. Nauk SSSR, 30, 301-305, 1941.

Kraichnan, R. H.: Inertial-range spectrum of hydromagnetic turbulence, Phys. Fluids, 8, 1385-1387, 1965.

Lavraud, B. and Cargill, P. J.: The magnetospheric cusps revealed: Results from the CLUSTER mission, Astronomy and Geophysics, 46, 1.32-1.35, 2005.

Lavraud, B., Dunlop, M. W., Phan, T. D., et. al.: Cluster observations of the exterior cusp and its surrounding boundaries under northward IMF, Geophys. Res. Lett., 29 (20), doi:10.1029/2002GL015464, 2002.

Lavraud, B., Phan, T. D., Dunlop, M. W., Taylor, M. G. G. T., Cargill, P. J., M.Bosqued, J., Dandouras, I., Réme, H., Sauvaud, J. A., Balogh, A., and Fazakerley, A.: The exterior cusp and its boundary with the magnetosheath under northward IMF: Cluster multi-event analysis, Ann. Geophys., 22, 3039-30554, 2004.

Lavraud, B., Fedorov, A., Budnik, E., Thomsen, M. F., Grikoriev, A., Cargill, P. J., Dunlop, M. W., Réme, H., Dandouras, I., and Balogh, A.: High-altitude cusp flow dependence on IMF orientation: A 3-year Cluster statistical study, J. Geophys. Res., 110, A02209, doi:10.1029/2004JA010804, 2005.

Le, G., Blanco-Cano, X., Russell, C. T., Zhou, X.-W., Mozer, F., Trattner, K. J., Fuselier, S. A., and Anderson, B. J.: Electromagnetic ion cyclotron waves in the high-altitude cusp: Polar observations, J. Geophys. Res., 106, 19 067-19079, 2001.

Leamon, R. J., Smith, C. W., Ness, N. F., and Matthaeus, W. H.: Observational constraints on the dynamics of the interplanetary magnetic field dissipation range, J. Geophys. Res., 103, 47754787, 1998.

Lysak, R. L. and Lotko, W.: On the kinetic dispersion relation for shear Alfvén waves, J. Geophys. Res., 101, 5085-5094, 1996.

Nykyri, K., Cargill, P. J., Lucek, E., Horbury, T. S., Balogh, A., Lavraud, B., Dandouras, I., and Rème, H.: Ion cyclotron waves in the high altitude cusp: CLUSTER observations at varying spacecraft separations, Geophys. Res. Lett., 30(24), 2263-2269, doi:10.1029/2003GL018594, 2003.

Nykyri, K., Cargill, P. J., Lucek, E. A., Horbury, T. S., Lavraud, B., Balogh, A., Dunlop, M. W., Bogdanova, Y., Fazarkerley, A., 
Dandouras, I., and Réme, H.: Cluster observations of magnetic field fluctuations in the high-altitude cusp, Ann. Geophys., 22, 2413-2429, 2004.

Paschmann, G., Haerendel, G., Sckopke, N., Rosenbauer, H., and Hedgecock, P. C.: Magnetic field characteristics of the distant polar cusp near local noon: the entry layer, J. Geophys. Res., 81, 2883-2899, 1976.

Penano, J. R. and Ganguli, G.: Generation of electromagnetic ion cyclotron waves in the ionosphere by localized transverse dc electric fields, J. Geophys. Res., 107 (A8), doi:10.1029/2001JA000279, 2002.

Rème, H., Aoustin, C., Bosqued, J. M., Dandouras, I., Lavraud, B., et. al.: First multispacecraft ion measurements in and near the Earth's magnetosphere with the identical Cluster ion spectrometry (CIS) experiment, Ann. Geophys., 19, 1303-1354, 2001.

Reynolds, M. A. and Ganguli, G.: Ion Bernstein waves driven by two transverse flow layers, Phys. Plasmas, 5, 2504-2512, 1998.

Rönnmark, K.: Waves in homogenous, anisotropic multicomponent plasmas (WHAMP), KRI, Tech. rep., 1982.

Russell, C. T., Le, G., and Petrinec, S. M.: Cusp observations of high-and low-latitude reconnection for northward IMF: An alternate view, J. Geophys. Res., 105, 5489-5495, 2000.

Sahraoui, F., Pincon, J., Belmont, G., Rezeau, L., CornilleauWehrlin, N., Robert, P., Mellul, L., Bosqued, J., Balogh, A., Canu, P., and Chanteur, G.: ULF wave identification in the magnetosheath: The k-filtering technique applied to Cluster II data, J. Geophys. Res., 108, 1-1, 2003.

Savin, S. P., Romanov, S. A., Fedorov, A. O., Zelenyi, L., Klimov, S. I., Yermolaev, Y. I., Budnik, E. Y., Nikolaeva, N. S., Russell, C. T., Zhou, X. W., Urguhart, A. L., and Reiff, P. H.: The cusp/magnetosheath interface on May 29, 1996: Interball-1 a nd Polar observations, Geophys. Res. Lett., 3015-3018, 1998.

Savin, S. P., Zelenyi, L., Maynard, N. C., Sandahl, I., Kawano, H., Russell, C. T., Romanov, S., Blecki, J., and et al.: MultiSpacecraft Tracing of Turbulent Boundary Layer, Adv. Space Res., 30(12), 2821-2830, 2002.
Savin, S. P., Zelenyi, L., Romanov, S. A., Sandahl, I., Pickett, J., Amata, E., Avanov, L., Blecki, J., et al.: Magnetosheath-cusp interface, Ann. Geophys., 22, 183-212, 2004.

Scudder, J. D., Mozer, F. S., Maynard, N. C., and Russell, C. T.: Fingerprints of collisionless reconnection at the separator, J. Geophys. Res., 107, SMP13-1 to SMP13-38, 2002.

Stasiewicz, K., Bellan, P., Chaston, C., Kletzing, C., Lysak, R., Maggs, J., Pokhotelov, O., Seyler, C., Shukla, P., Stenflo, L., Streltsov, A., and Wahlund, J.-E.: Small Scale Alfvénic Structure in the Aurora, Space Sci. Rev., 92, 423-533, 2000.

Sundkvist, D., Vaivads, A., André, M., Wahlund, J.-E., Hobara, Y., Joko, S., Krasnoselskikh, V. V., Bogdanova, Y. V., Buchert, S. C., Cornilleau-Wehrlin, N., Fazakerley, A., Hall, J.-O., Rème, H., and Stenberg, G.: Multi-spacecraft determination of wave characteristics near the proton gyrofrequency in high-altitude cusp, Ann. Geophys., 23, 983-995, 2005.

Twitty, C., Phan, T. D., Paschmann, G., Lavraud, B., Rème, H., and Dunlop, M.: Cluster survey of cusp reconnection and its IMF dependence, Geophys. Res. Lett., 31, L19808, doi:10.1029/2004GL020646, 2004.

Vontrat-Reberac, A., Bosqued, J. M., Taylor, M. G., Lavraud, B., Fontaine, D., Dunlop, M. W., Laakso, H., CornilleauWerhlin, N., Canu, P., and Fazarkerley, A.: Cluster observations of the high-altitude cusp for northward interplanetary magnetic field: a case study, J. Geophys. Res., 108(A9), doi:10.1029/2002JA001717, 2003.

Zhou, X. W., Russell, C. T., Le, G., Fuselier, S. A., and Scudder, J. D.: The Polar cusp location and its dependence on dipole tilt, Geophys. Res. Lett., 26, 429-432, 1999.

Zhou, X. W., Russell, C. T., Le, G., Fuselier, S. A., and Scudder, J. D.: Solar wind control of the polar cusp at high altitude, J. Geophys. Res., 105, 245-251, 2000. 